\title{
Magnetically actuated self-clearing catheter for rapid in situ blood clot clearance for improved hemorrhagic stroke treatment
}

\section{Qi Yang}

Purdue University West Lafayette https://orcid.org/0000-0003-4613-7582

\section{Angel Enriquez}

Purdue University West Lafayette

\section{Dillon Devathasan}

Purdue University

\section{Craig Thompson}

Purdue University

\section{Dillan Nayee}

Purdue University

\section{Ryan Harris}

Purdue University

\section{Douglas Satoski}

Purdue University

\section{Barnabas Obeng-Gyasi}

Purdue University

\section{Albert Lee}

Goodman Campbell Brain and Spine

\section{R. Timothy Bentley}

Purdue University

Hyowon Lee ( $\nabla$ hwlee@purdue.edu )

Purdue University West Lafayette https://orcid.org/0000-0001-7628-1441

\section{Article}

Keywords: self-cleaning catheter, in situ blood clot clearance, hemorrhagic stroke treatment

Posted Date: April 23rd, 2021

DOI: https://doi.org/10.21203/rs.3.rs-339235/v1 
License: (c) (i) This work is licensed under a Creative Commons Attribution 4.0 International License. Read Full License

Version of Record: A version of this preprint was published at Nature Communications on January 26th, 2022. See the published version at https://doi.org/10.1038/s41467-022-28101-5. 


\title{
Magnetically actuated self-clearing catheter for rapid in situ blood clot clearance for improved hemorrhagic stroke treatment
}

\author{
Qi Yang ${ }^{1,2,3}$, Angel Enriquez ${ }^{1,2}$, Dillon Devathasan ${ }^{4}$, Craig A. Thompson ${ }^{4}$, Dillan Nayee ${ }^{1}$, \\ Ryan Harris ${ }^{1}$, Douglas Satoski ${ }^{1}$, Barnabas Obeng-Gyasi ${ }^{1}$, Albert Lee ${ }^{5}$, R. Timothy \\ Bentley $^{4}$, and Hyowon Lee ${ }^{\star 1,2}$
}

\author{
${ }^{1}$ Weldon School of Biomedical Engineering \\ ${ }^{2}$ Birck Nanotechnology Center \\ ${ }^{3}$ School of Electrical and Computer Engineering \\ ${ }^{4}$ College of Veterinary Medicine \\ ${ }^{5}$ Goodman Campbell Brain and Spine, \\ $1,2,3,4$ Purdue University, West Lafayette, IN 47907, USA \\ 5 Indianapolis, IN 46202 USA \\ *To whom correspondence should be addressed; E-mail: hwlee@purdue.edu.
}

\begin{abstract}
Maintaining the patency of indwelling drainage devices is critical in preventing further complications following an intraventricular hemorrhage (IVH) and other chronic disease management. Surgeons often use drainage devices to remove blood and cerebrospinal fluid but these catheters frequently become occluded with hematoma. Using an implantable magnetic microactuator, we created a self-clearing catheter that can generate large enough forces to break down obstructive blood clots by applying time-varying magnetic fields. In a blood-circulating model, our self-clearing catheters demonstrated a $>7 x$ longer functionality than traditional catheters (211 vs. $27 \mathrm{~min}$ ) and maintained a low pressure for longer periods (239 vs. $79 \mathrm{~min})$. Using a porcine IVH model, the self-clearing catheters showed a greater survival rate than control catheters $(86 \%$ vs. $0 \%)$ over the course of 6 weeks. The treated animals also had significantly smaller ventricle sizes 1 week after implantation compared to the control animals with traditional catheters. Our results suggest that these magnetic microactuator-embedded smart catheters can expedite the removal of blood from the ventricles and potentially improve the outcomes of critical patients suffering from often deadly IVH.
\end{abstract}

\section{Introduction}

Brain hemorrhage is one of the most common and lethal forms of stroke, affecting more than 2 million patients annually worldwide. In $45 \%$ of these cases, bleeding occurs inside the ventricles of the brain and leads to intraventricular hemorrhage (IVH). When the blood clot (hematoma) forms and obstructs the circulation of cerebrospinal fluid (CSF), IVH can lead to an even deadlier condition (50-80\% mortality) known as post-hemorrhagic hydrocephalus ( $\mathrm{PHH}, 40 \%$ of IVH) $)^{1,2}$. IVH is especially common in preterm pediatric patients with low birthweight, and subsequent PHH can have devastating neurodevelopmental consequences ${ }^{3}$.

A gold-standard in the treatment of IVH is the rapid removal of hematoma to prevent further deterioration using various interventional methods including open surgery, minimally-invasive catheter-based drainage, and thrombolytic agents. These are used to relieve the elevated intracranial pressure (ICP), restore CSF flow, and minimize blood exposure to the ventricles ${ }^{4,5}$. For most IVH patients, external ventricular drainage (EVD), ventricular reservoir devices, or neuroendoscopy are used to remove blood-filled CSF and stabilize increasing $\mathrm{ICP}^{4,6}$.

Unfortunately, there are conflicting reports on the clinical efficacy of drainage devices and there is no established guideline to help determine the proper conditions for EVD implantation ${ }^{7}$. This limitation may partly be due to the difficulty in maintaining the patency of the drainage device in the blood-filled ventricle ${ }^{8,9}$. To facilitate the blood clot removal, thrombolytic agents are sometimes used with a drainage device for increased clot resolution ${ }^{10}$. However, using fibrinolytic agents on hemorrhagic patients is a highly controversial topic due to the elevated risk of additional bleeding and their unknown long-term effects ${ }^{11,12}$.

The obstruction of drainage devices is a notorious issue recognized for more than half a century ${ }^{13}$. To maintain the patency in drainage systems, catheter flushing often takes place in intensive care units ${ }^{14}$. However, flushing alone is often ineffective in clearing the hematoma, which leads to several replacements of failed drainage devices that also increase the risk of infection and 
other complications including ventriculitis ${ }^{15-17}$. The high obstruction rate of these critical medical devices results in significant complications when treating IVH, $\mathrm{PHH}$, and other applications where indwelling catheters are needed ${ }^{12}$.

There are efforts to combat the occlusion issues in EVD and other drainage devices. For example, researchers have investigated the use of dual catheters in high volume $\mathrm{IVH}^{18,19}$. There are also reports of using high-intensity focused ultrasound as a way to dissolve clots in $s i t u^{20-23}$. However, increasing the number of catheters also increases the risk of infection and the potential concerns of off-target tissue damage remain for the ultrasound-induced clot reduction.

Magnetic microactuators have previously shown to be effective in vitro in removing molecular and cellular scale biofouling materials on-demand using remote application of time-varying magnetic fields ${ }^{24-26}$. Moreover, there are now examples of magnetically actuated microrobots for various in vivo clinical applications including drug delivery ${ }^{27-30}$, stem cell transplantation ${ }^{31}$, and minimally invasive surgeries ${ }^{32-34}$. These magnetically-powered micro- and nano-scale transducers provide a significant advantage over conventional medical devices because they allow implanted devices to be manipulated in situ without the need for additional surgical intervention using externally applied magnetic fields ${ }^{35}$.

In this work, we present a novel self-clearing implantable catheter that is enabled by microscale magnetic actuators. These externally controlled magnetic microactuators can rapidly breakdown intraventricular thrombosis with its large-deflection actuation to maintain patency in implantable catheters. Using in vitro and in vivo experiments, here we show that these self-clearing smart catheters with magnetic microactuators can help reduce the size of the obstructive hematoma, improve drainage device reliability, and increase the survival of IVH-induced animals.

\section{Results}

\section{Device design and characterization}

To fabricate the implantable magnetic microactuator for the prevention of hematoma accumulation, we applied conventional surface micromachining techniques as described in the Methods section. Figure 1a-f briefly shows the fabrication process. Fig. 1g highlights the two microactuator designs. The dimensions of each design are listed in Supporting Information Table 1 and 2. The first design is a simple rectangular cantilever we have previously reported ${ }^{25,36}$.

The new design features a serpentined flexure with tear-drop shaped ferromagnetic elements (Figure. 1g). The serpentine flexure consists of 4 windings with $5,400 \mu \mathrm{m}$-long straight segments connected by 4 arcs providing $100 \mu \mathrm{m}$ gaps in between them. Similar serpentine flexures have been reported for making ultra low-frequency resonator and piezoelectric energy harvest devices ${ }^{37-40}$. The serpentine flexure provides a 6-times smaller bending stiffness and enhanced deflection within a limited footprint. In addition to improved beam design, the large aspect ratio magnetic elements provide additional magnetic torque compared to the previous versions despite having nearly identical volume because of magnetic geometry anisotropy. A detailed description of the magnetic element dimensions and the analytical evaluation of magnetic torque is in the supplemental information. The microfabricated thin-film microactuators were then integrated into a custom catheter to create a self-clearing ventricular catheter (Fig 1g). Fig. 1h illustrates a typical actuation motion of a microactuator with serpentine flexure (SI Video 1). In the presence of time and spatially varying magnetic fields, the actuator deflects in and out of the plane for a more dynamic hematoma removal (SI Video 2).

Fig. 2 demonstrates the improvements of the new magnetic microactuator over the straight beam design. We characterized the magnetic properties of the electroplated ferromagnetic elements using a magnetometer to calculate the magnetic torque assuming a field-dependent, non-saturated magnetization (SI Fig. 1-3). Fig. 2a-b show that the magnetic torque varies as the function of applied magnetic field angle $\theta$ and that maximum torque can be achieved at $40^{\circ}$. We saw as much as $75 \%$ improvement when the field angle $\theta$ equals $40^{\circ}$.

We found that the new flexure design improved actuator displacement per applied magnetic field strength. The numerical analysis showed a larger deflection and smaller stress on the serpentine flexure than the straight beam when a vertical point load was applied to its tip (Fig. 2c). Fig. 2d-e shows varying degrees of deflection and corresponding maximum stress on the beam at various load ranges. We saw that the serpentine flexure provides twice as much displacement over the straight beam over the same loading condition. The maximum static stress calculated for serpentine flexure was $36 \mathrm{MPa}$ at $0.1 \mathrm{mN}$, which is significantly lower than the tensile stress of this polyimide (131 MPa).

The $0.1 \mathrm{mN}$ loading was chosen from the magnetic resonant (MR) safety perspective ${ }^{41}$. A typical human whole-body MR system produces a maximum spatial gradient between $10-50 \mathrm{mT} / \mathrm{m}^{42-44}$. Assuming the ferromagnetic element was fully saturated, the $0.1 \mathrm{mN}$ load corresponds to the magnetic force produced from a gradient of $4 \mathrm{~T} / \mathrm{m}$, an order of magnitude greater than the physical constraint. Therefore, it is unlikely that our device will be damaged due to mechanical deformation in an MR system.

The improvements in magnetic torque and mechanical compliance of the flexure allow the new microactuator to achieve a greater deflection per given magnetic flux density (Fig. 2f). Although the previous microactuators were effective against protein and cellular biofouling 25,45 , those were not effective against macroscopic hematoma. Therefore, our primary objective was to increase the magnetic torque and rapid displacement to better remove a more robust thrombotic mass that plagues 

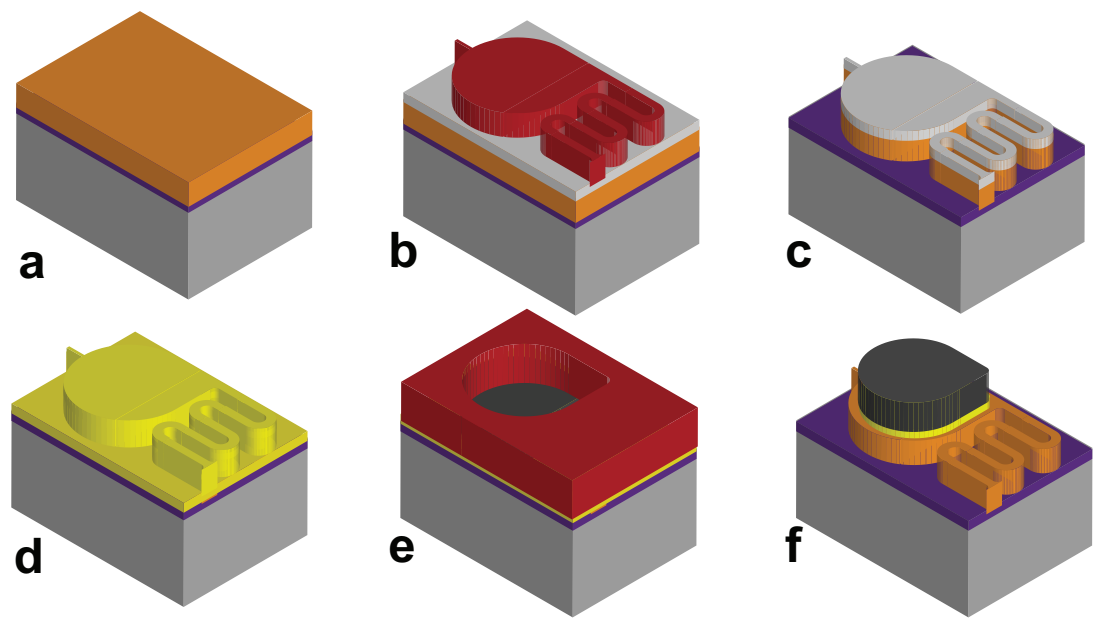

Photoresist

\section{Polyimide}

\section{$\mathrm{Cr} \quad \mathrm{Ni}$}

$\mathrm{Au}$

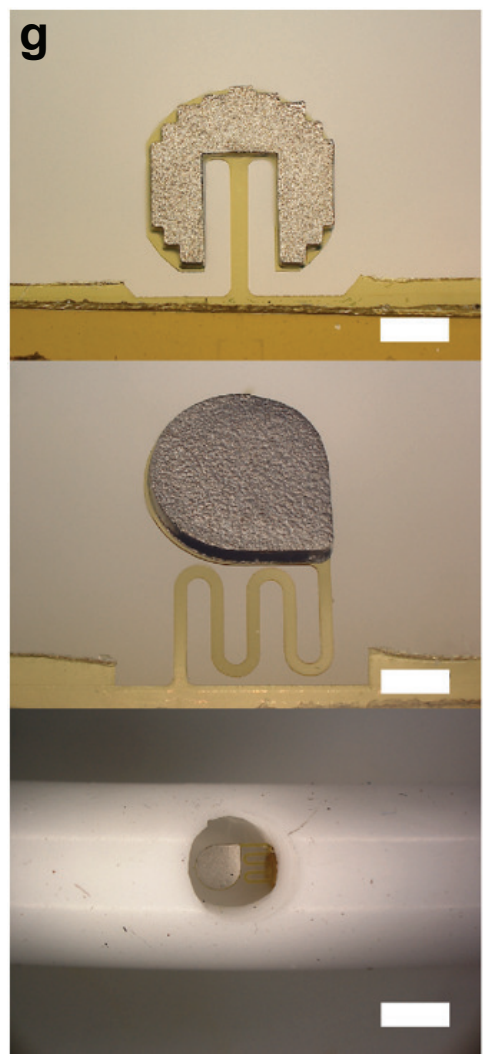

h
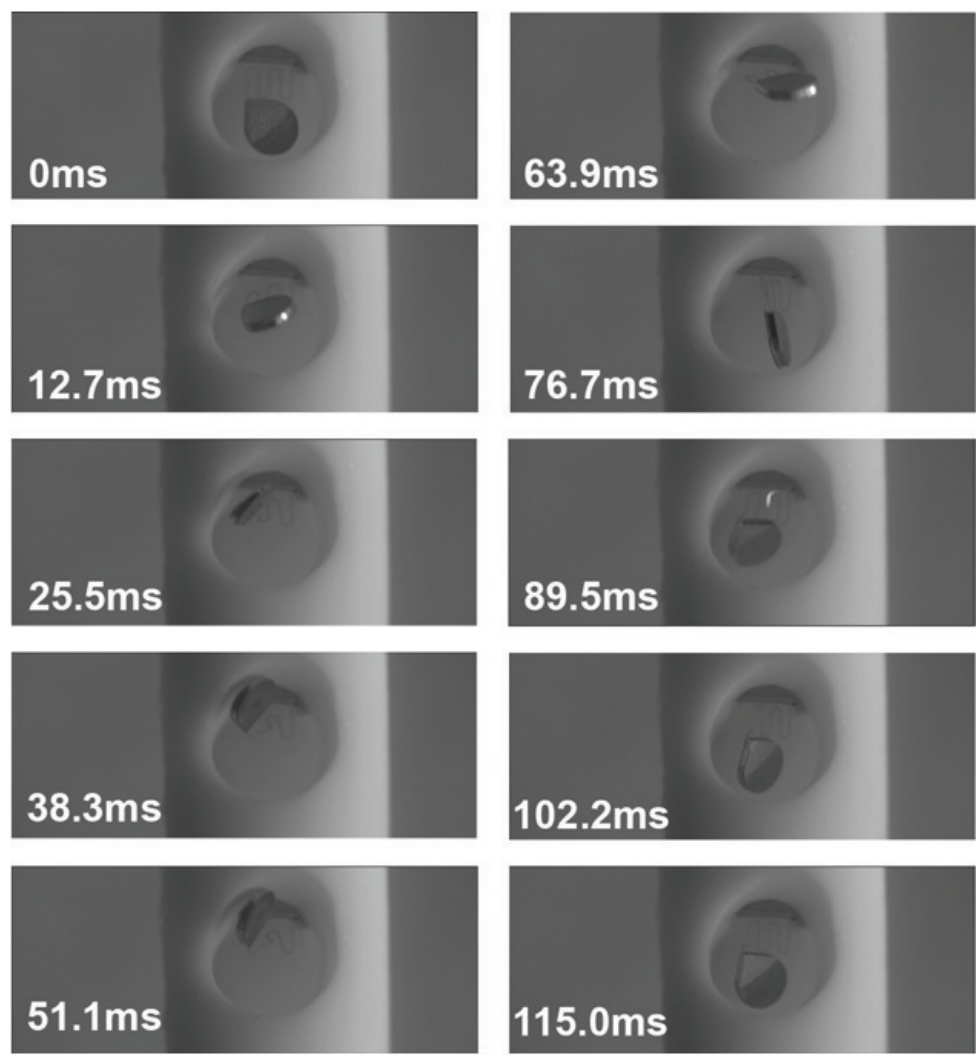

Figure 1. Design and fabrication of a self-clearing ventricular catheter. (a) Polyimide spin coat and curing. (b) Evaporation of $\mathrm{Cr}$ etching mask and define photoresist for actuator outline. (c) Wet etch of $\mathrm{Cr}$ and dry etch of polyimide. (d) Removal of $\mathrm{Cr}$ and evaporation of $\mathrm{Au}$ as a conduction layer. (e) application of photoresist and $\mathrm{Ni}$ electroplating. (f) removal of photoresist and remaining Au layer. (g) Optical images of fabricated magnetic actuators of straight type (top) and serpentine type (middle). Scale bars $=325 \mu \mathrm{m}$. A catheter with an integrated actuator (bottom). Scale bar $=1 \mathrm{~mm}$. (h) A typical motion of actuation in deionized (DI) water for one actuation period.

drainage devices in IVH patients. Our results show that we were able to achieve this goal. The new microactuator achieved $>80^{\circ}$ deflection with $15 \mathrm{mT}$ compared to only $30^{\circ}$ for the previous design. Our dynamic response analysis demonstrates that $15 \mathrm{~Hz}$ of actuation frequency produces the largest deflection and highest speed of the actuator (SI Fig. 3a-c). 
a
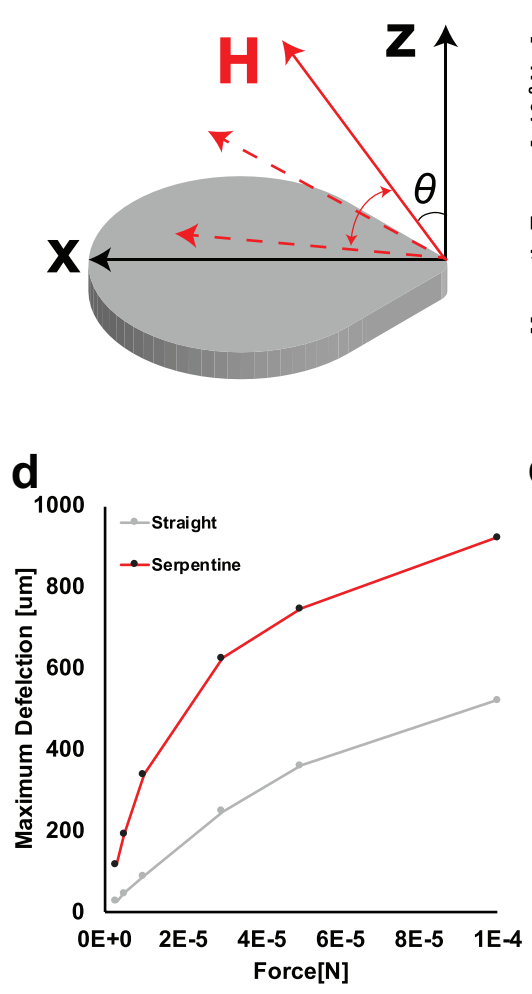

b

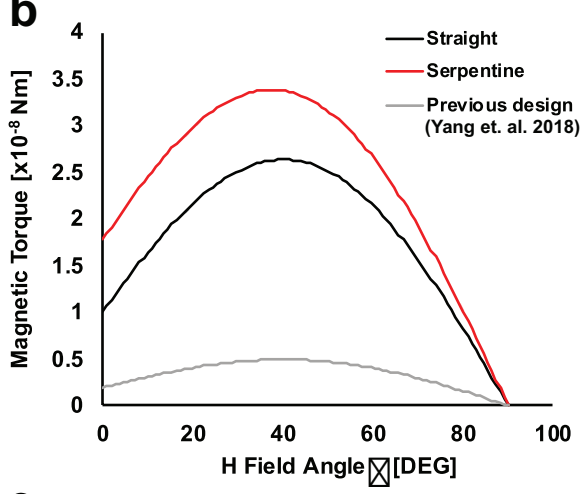

e

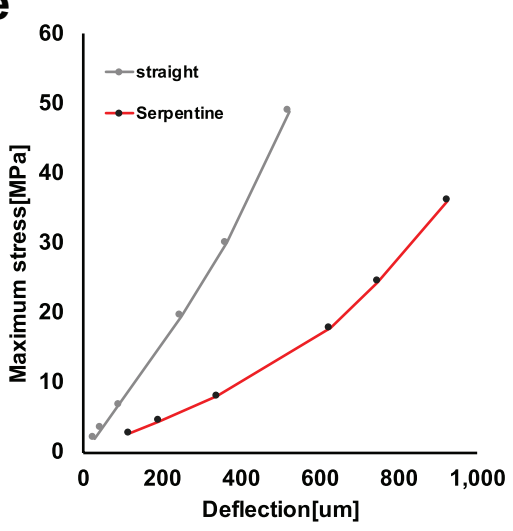

C

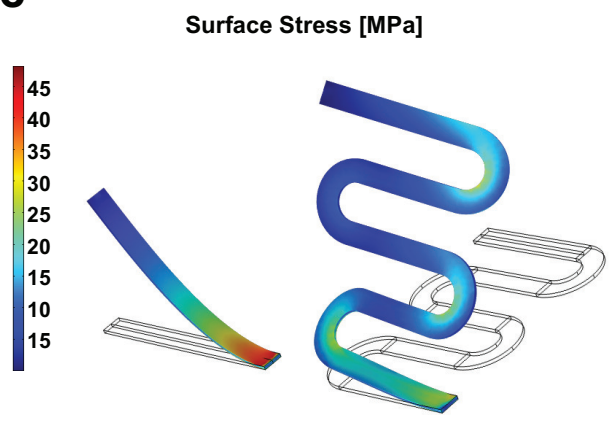

f

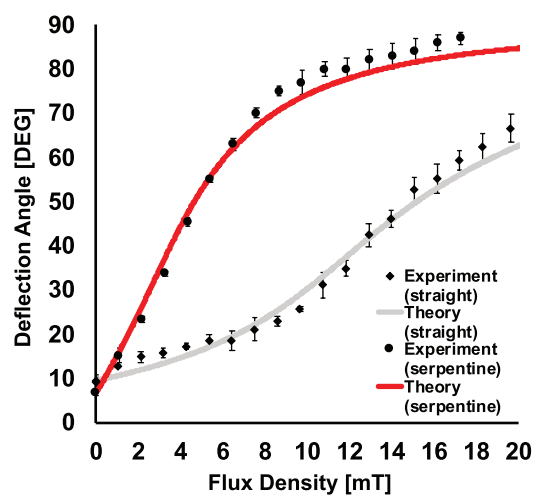

Figure 2. Mechanical characterization. (a) Definition of the coordinate system and magnetic field angle $\theta$. (b) Calculated magnetic torque produced on the ferromagnetic element of serpentine (red line) and straight actuator (black line) under 15mT at different field angles. (c) Finite element analysis of stress distribution on cantilevers' surfaces under $0.1 \mathrm{mN}$ load at the tip. (d) Maximum calculated deflection under loading condition $(0.01$ to $0.1 \mathrm{mN})$ for both cantilever type from finite element simulation. (e) Maximum calculated stress under various deflection conditions for both cantilever type from finite element simulation. (f) Static deflection angle prediction and measurements for the serpentine and the straight flexures. Data are expressed as mean \pm standard deviation $(n=3)$.

\section{In vitro evaluation}

To demonstrate the blood-clot removal capabilities of our self-clearing catheters, we developed an in vitro circulation system ${ }^{46}$. Although several groups have reported a reduction of hematoma mass using a static condition ${ }^{21,23}$, none have demonstrated an effective clot prevention capability in a continuous flow environment, which is more physiologically-relevant for our target application.Fig. 3a shows the experimental setup, which mimics a fixed-volume ventricle that circulates sanguineous phosphate buffered solution (PBS) as a CSF substitute. Using a peristaltic pump, we pumped diluted porcine blood (50:50 with PBS) out of the sealed chamber through different catheter designs. The blood-PBS mixture ratio was determined experimentally to reliably produce hematoma within $4 \mathrm{~h}$. As the fluid flowed through the circulation system, we applied a time-varying magnetic field on all 3 groups: control, flushing, and self-clearing catheters (SI Fig. 4). The flushed catheters were identical to control catheters but had a three-way stopcock valve attached to the line of the drainage device with one connector having a syringe with $2 \mathrm{~mL}$ of PBS. Once $5 \mathrm{~min}$ of pressure above the established threshold $(40 \mathrm{mmHg}$ ) was reached, the valve was redirected to allow flow from the syringe, and the saline injection was introduced. After the full saline injection, flow in the circulation system was re-established. Flushing was repeated as many times as necessary in the $4 \mathrm{~h}$ experiment.

In general, the self-clearing catheters with integrated microactuators exhibited a smaller hematoma mass over their inlet pores compared to the flushing and control catheters (Fig. 3b). We also analyzed the effect of magnetic microactuation on the hematoma by analyzing the structure using an SEM (Inset Fig. 3b). Utilizing a grading scale developed and analyzed by a board-certified clinical pathologist, we found that the fibrin mesh network significantly differed between the control and treatment devices $(p<0.001$, SI Fig. 5). The fragments from the treatment device displayed almost no fragments of fibrin, further confirming that our self-clearing catheters can break apart hematoma to allow drainage through the ventricular catheter.

We quantified the impact of magnetic microactuation by measuring the differential pressure between the inlet and the outlet 

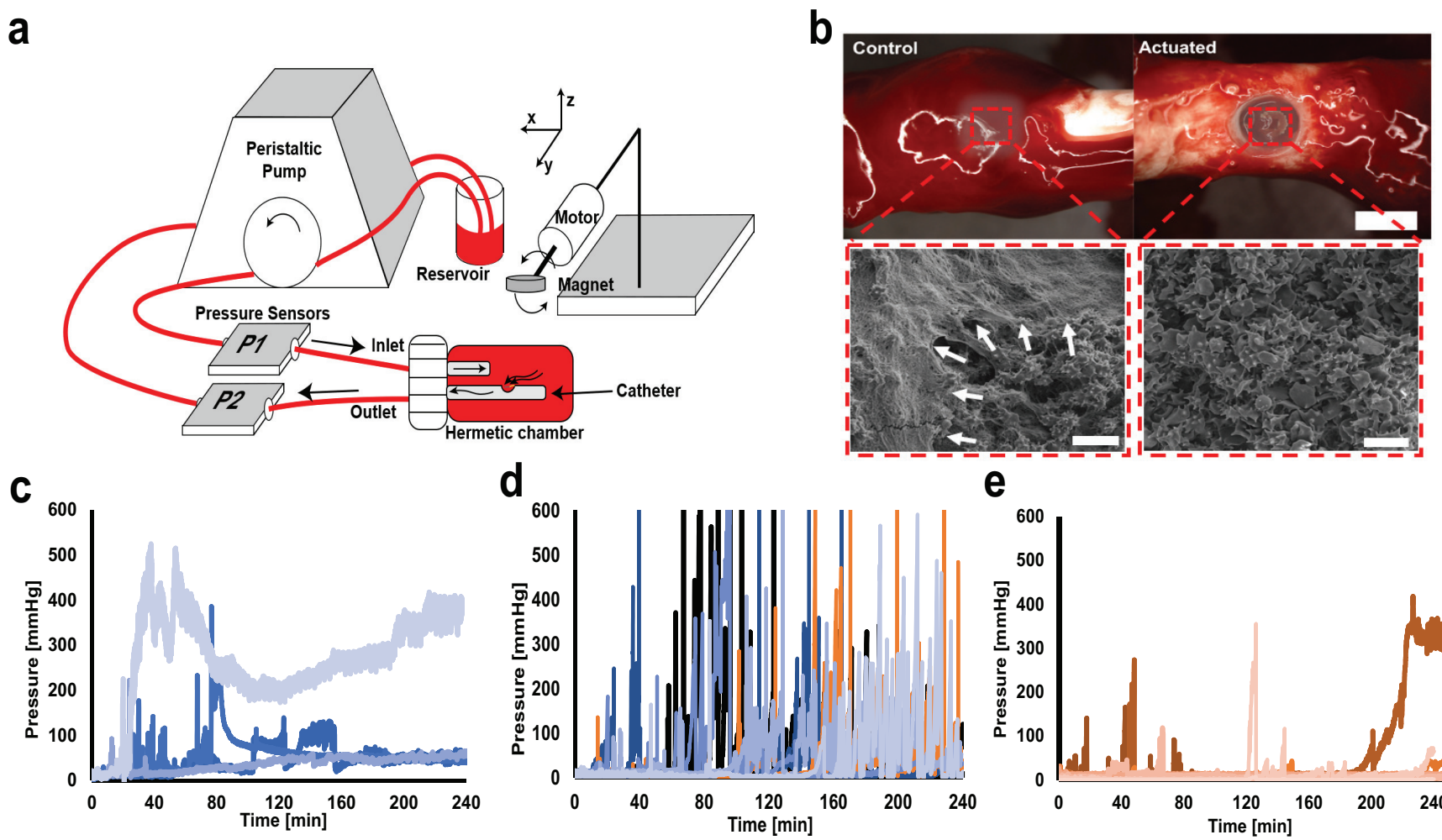

e
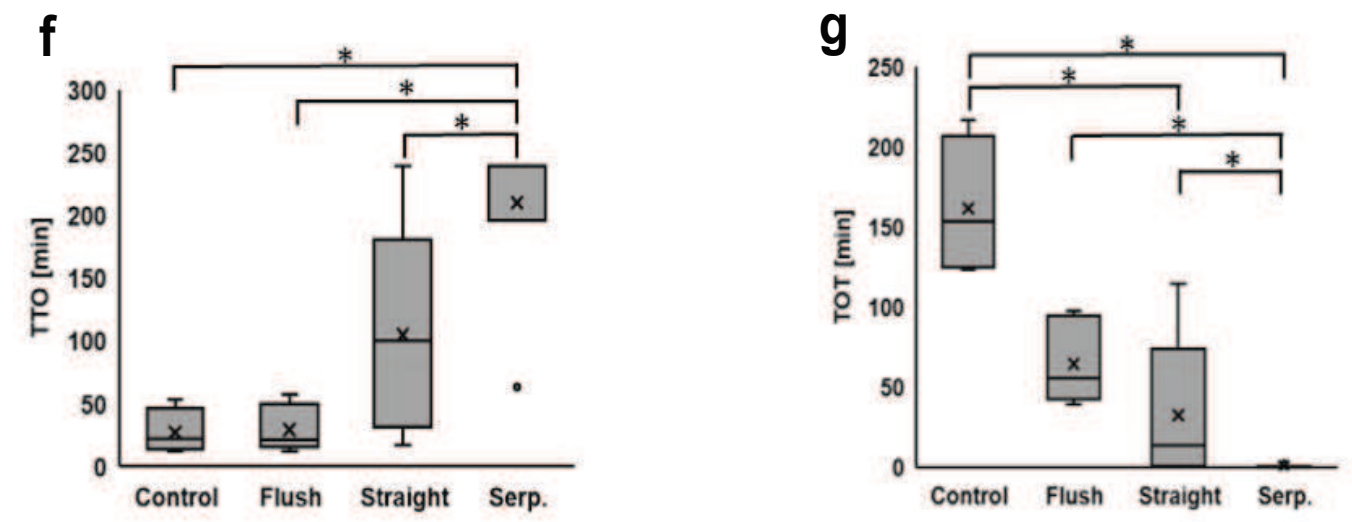

Figure 3. In vitro experimental setup and results. (a) Schematic of the bench-top blood circulation and magnetic actuation setup. (b) A representative visual comparison of control vs. actuated self-clearing catheter at the conclusion of the experiment. Scale bar $=1.5 \mathrm{~mm}$. Inset: SEM images obtained from hematoma fragments of control group. White arrows depict the robust fibrin network left unscathed and covering red blood cells. The hematoma fragments of the actuating group lack the fibrin network surrounding the red blood cells. Scale bar $=10 \mu \mathrm{m}$. (c) Differential pressure (P2-P1) recording of all control catheters $(n=4)$. (d) Differential pressure $(P 2-P 1)$ recording of all flushing catheters $(n=6)$. Note the frequent pressure spikes associated with each flushing. (e) Differential pressure (P2-P1) recording of all self-clearing catheters $(\mathrm{n}=11)$. The figure includes data from 5 straight and 6 serpentine devices. (f) Comparison of time-to-occlusion (TTO) to reach $40 \mathrm{mmHg}$ between the control catheter, catheters that underwent flushing, and self-clearing catheters. There were two versions of self-clearing catheters with either straight vs. serpentine flexure microactuators. One-way ANOVA with Tukey post-hoc analysis showed overall statistical significance between groups. Individual comparison and significance $(p<0.01)$ are indicated with *. $(\mathrm{g})$ Comparison of the total time over the threshold (TOT). One-way ANOVA with Tukey post-hoc analysis confirmed statistical significance between groups. Individual comparison and significance $(p<0.01)$ are indicated with *. 
during the experiment. Fig. 3c-e show differential pressure throughout the experiments. Without any blood in circulation, the differential pressure ranged between $5-15 \mathrm{mmHg}$, which is comparable to the normal intraventricular pressure of a patient. However, with blood, the control catheters exhibited an exceptionally high average pressure of $100 \pm 111 \mathrm{mmHg}$, and the flushing catheters had an average pressure of $41 \pm 10 \mathrm{mmHg}$, whereas the self-clearing catheters had an average pressure of $11 \pm 31 \mathrm{mmHg}$. To compare all catheter groups, a threshold pressure of $40 \mathrm{mmHg}$ was chosen because intracranial pressure (ICP) exceeding this value is considered life-threatening ${ }^{47-49}$. The time-to occlusion (TTO) was defined by the time to reach this threshold. We postulate that TTO indicates how quickly the drainage system shows functional deterioration due to hematoma. For control catheters, the average TTO was $27 \pm 18$ min. For flushing catheters, the average TTO was $29 \pm 18$ min, which is similar to the control catheters. This was expected as both devices are single pore catheters without the actuator. For the treatment group, the average TTO was delayed to $104 \pm 86$ min for straight devices and $211 \pm 72$ min for serpentine devices.

We also determined the total time over the threshold pressure of $40 \mathrm{mmHg}$ (TOT). We postulated that the TOT indicates the resilience of the drainage system to combat blood-clot induced failure. The average TOT for control devices was $162 \pm 44$ min during a 240 min experiment. The average TOT for the flushing catheters was $64 \pm 25$. In comparison, the average TOT was $33 \pm 48 \mathrm{~min}$ for the catheters with straight devices and $0.3 \pm 0.8 \mathrm{~min}$ for the catheters with serpentine devices. Fig. $3 \mathrm{f}-\mathrm{g}$ compares the TTO and TOT for each condition.

In all 4 control catheters, we saw that the differential pressure remained above the threshold at the end of the circulation period, indicating robust and sustained obstruction by a hematoma (Fig. 3c). The catheters used for the flushing test had 3 out of 6 that remained above the pressure threshold, indicating large hematoma in the system was still present and was not absolved by the flushing. The average number of flushes per experiment run $(n=6)$ was $8 \pm 3$ resulting in high pressure spikes outside of the pressure range of interest throughout the experiment time. Conversely, 8 out of 11 self-clearing catheters (5 straight and 6 serpentine devices) exhibited relatively lower pressure $(<20 \mathrm{mmHg})$ at the end of the circulation (Fig. 3e). Compared to the control catheters, we also observed less frequent pressure spikes.

These results demonstrate that our self-clearing catheters can significantly delay occlusion due to hematoma and improve device reliability. Our experiments also show the cumbersome nature of having an experienced clinician be vigilant for obstruction and applying timely flushing with a risk of pressure spikes. In comparison, our self-clearing catheter can easily be actuated using external control with minimal risk for pressure spikes. Moreover, these results confirmed our hypothesis that the larger actuation deflection afforded by the serpentine flexure design could improve blood-clot removal capabilities. We suspect that the rapid translational motion of our microactuators causes localized shear at the catheter inlet, which exceeds the threshold above which hematoma becomes incapable to attach and accumulate on the catheter surface ${ }^{50-52}$. In future work, we may be able to use numerical evaluation to estimate the amount of shear stress our microactuators generate in a circulating flow environment.

\section{In vivo evaluation}

To verify the results from our in vitro experiment, we developed a porcine model of IVH and evaluated the impact of self-clearing catheters in vivo (SI Fig. 8). Initially, we performed preliminary studies with 6 pigs to determine the amount of blood to be injected to cause large hematoma in the ventricle and sustained intracranial hypertension (SI Fig. 9). As a result of these studies, we identified $10 \mathrm{ml}$ of blood admixed with 140 units of thrombin immediately prior to injection as a reliable way to cause IVH and subsequent PHH. The blood and thrombin were injected into the right lateral ventricle in 3 equal aliquots. The timeline of the in vivo evaluation can be seen in Fig. 4.

A total of 13 pigs were used for the evaluation. The median weight was $28.0 \pm 3.9 \mathrm{~kg}$. The baseline ICP was $11 \pm 5.2$ $\mathrm{mmHg}$. As the blood-thrombin injection was paused whenever ICP was $\geq 50 \mathrm{mmHg}$, the injection was performed over 2 to $15 \mathrm{~min}$ (median, $8 \mathrm{~min}$ ). During injection of the third and final aliquot of blood, ICP would typically immediately increase above $50 \mathrm{mmHg}$ with each injection of about $1 \mathrm{ml}$, then quickly fall (SI Fig. 10). The ventricular catheter was placed $7.5 \pm 7.0$ min after the blood-thrombin injection was completed. A custom ventricular catheter with a single inlet pore was again used. ICPs reached a high but variable peak, fell quickly initially, then slowly declined to reach a plateau of around $13 \pm 4$ $\mathrm{mmHg}$. The custom ventricular catheter was connected to a conventional one-way valve and peritoneal catheter to form a ventriculoperitoneal (VP) shunt. The fully implanted system was used in place of conventional EVD to mitigate the potential risk of surgical site infection and to allow free movement for the recovered animals.

In all pigs, we used post-operative computed tomography (CT) to confirm the accurate intraventricular injection of blood and air. The post-shunting CT also confirmed the correct location of the ventricular catheter. In 1 Control and 1 Treatment pig, we determined that the only inlet pore had passed through the lateral ventricle and into the brain parenchyma. In both of these pigs, we performed a brief second surgery to retract the ventricular catheter and the subsequent CT confirmed the correct ventricular placement of the inlet pore.

All 6 Control pigs suffered sudden neurological decline and were found to have shunts obstructed with hematoma at necropsy. The sudden neurological decline in the Control pigs occurred $12 \mathrm{~h}$ to 5 days post-operatively (median, 3 days). 


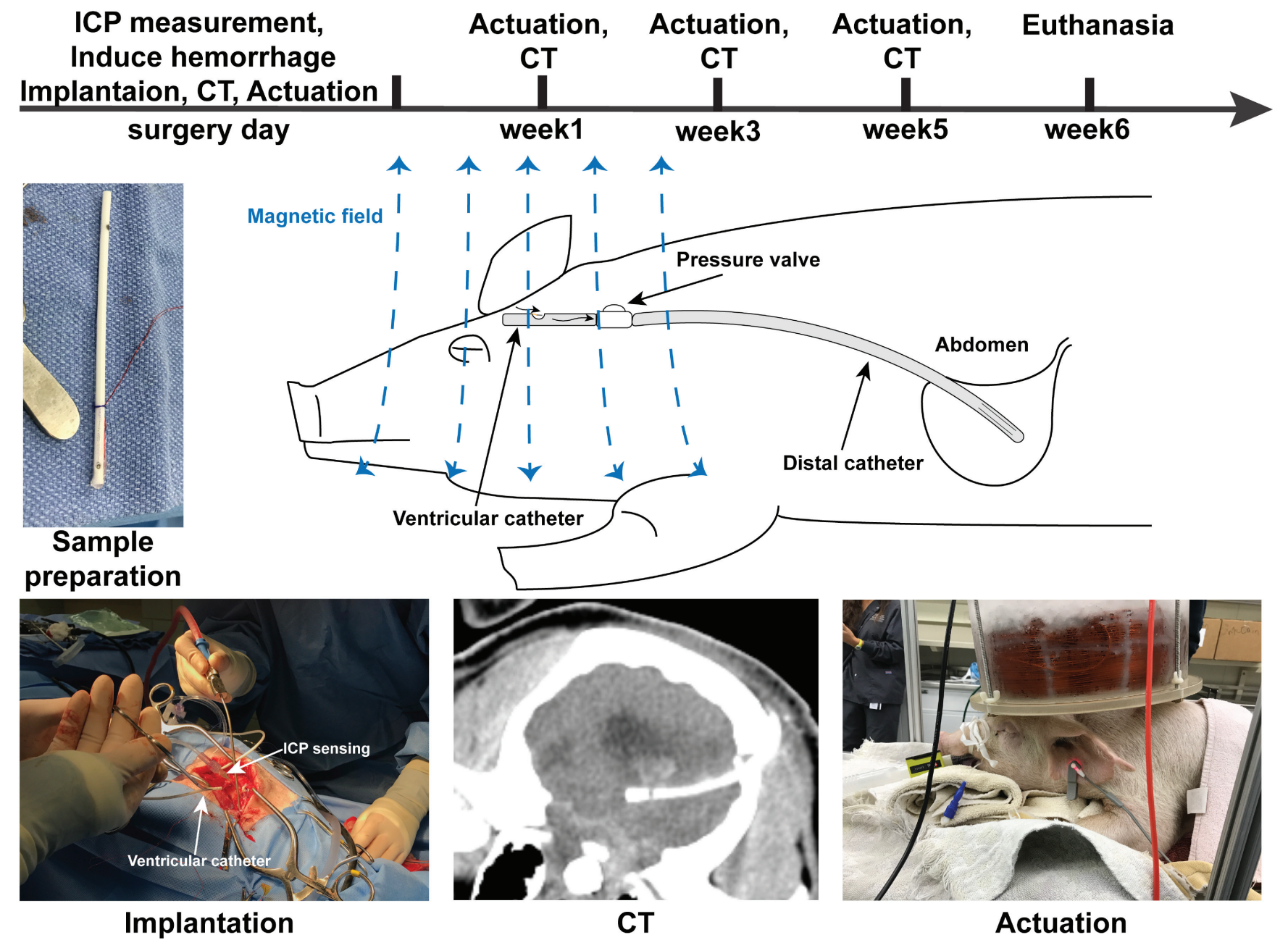

Figure 4. In vivo experimental timeline. The entire study is designed to lasts 6 weeks. On surgery day, we induced IVH and implanted a VP shunt. CT was performed before and after the surgical procedure, to measure the ventricular size and confirm ventricular catheter placement. Magnetic actuation (30 min) was performed for the Treated group immediately afterward. At weeks 1,3 , and 5, each surviving animal was subjected to $30 \mathrm{~min}$ of magnetic field and CT scans. All surviving animals were terminated at week 6 for explantation and necropsy.

Four of these animals became moribund within hours of the first sign of deterioration, and were euthanized. Post-mortem CT revealed marked enlargement of the ventricles since the post-operative $\mathrm{CT}$, transtentorial with or without cerebellar herniation, and continued correct ventricular location of the ventricular catheter without shunt displacement (Fig. 5a). The other 2 pigs showed a sudden, severe but non-fatal neurological decline. The ventricles were markedly larger on the next CT, and for the rest of the study. All explanted VP shunts in the control group showed evidence of hematoma inside their lumen (SI Fig. 11). There were a total of 8 hematomas in these 6 VP shunts (3 ventricular catheters, 3 valvular, 2 distal catheters).

On the contrary, only 1 of the 7 Treatment pigs suffered neurological decline and was found to have a complete shunt obstruction with hematoma at necropsy. This animal deteriorated $12 \mathrm{~h}$ post-operatively, rapidly became moribund, and was euthanized. This was the pig that had had a second surgery to slightly retract the ventricular catheter, resulting in delayed actuation. Upon post-mortem examination, we found further enlargement of the ventricles and persistence of intraventricular hematoma. Hematoma blocking the push connector (inlet for the valve) was found at necropsy (SI Fig. 12a). The valve itself was filled with sanguineous CSF but was not obstructed. This was the only fatal hematoma in the 7 VP shunts of the Treatment pigs. The ventricular catheters, valves, and distal catheters were otherwise patent without any evidence of obstructive hematoma (SI Fig. 12b). These observations suggest that magnetic actuation may be able to preserve the patency of the downstream drainage path despite being at the proximal end of the ventricular catheter. 


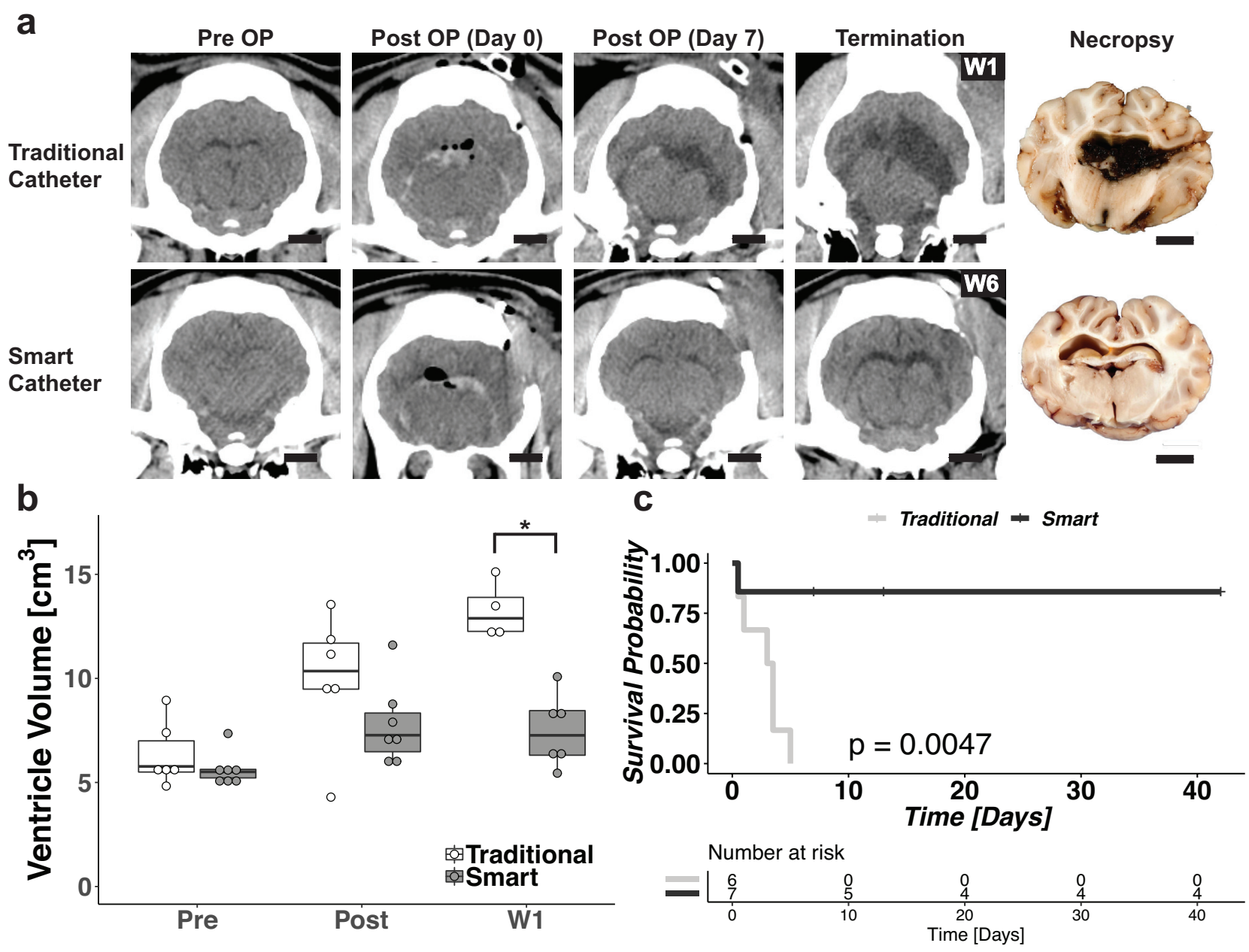

Figure 5. In vivo evaluation results. (a) Comparison of $\mathrm{CT}$ scans over the course of implantation for animals with traditional catheters (Control) and smart self-clearing catheters (Treated). Note the difference in ventricular volume before and after the surgery. There are signs of air pockets following the surgery (Post Op). After a week, the air pockets are resorbed but there is significant evidence of ventriculomegaly and hematoma in Control animals. The CT scan of the Control animal shows a significantly larger ventricle filled with hematoma compared to the Treatment animal. The photograph at necropsy also shows the enlarged ventricles. Scale bars $=1 \mathrm{~cm}$. (b) Box plot of ventricle volume until week 1 . The extended ventricle volume plot is available in SI Fig. 6. ANOVA indicated a significant difference in ventricle volume when treated with smart self-clearing catheters. Control animals with traditional catheters had a significant increase in ventricle volume by W1 $(p<0.001)$. (c) Kaplan-Meier survival plot with corresponding risk table. By week 1, the traditional shunt systems in all Control animals had failed whereas $80 \%$ of the shunt systems with self-clearing catheters remained obstruction-free with biweekly actuation.

SI Fig. 7 shows the survival plot when infections are counted as failures.

Infection One Control pig suffered from non-fatal shunt obstruction with hematoma on day 4, followed by dehiscence of the wound edges, post-operative infection, and euthanasia. Two Treatment pigs developed a fatal infection. One was euthanized on day 7 for peri-operative infection. The other rubbed his head on cage bars post-operatively. The skin edges dehisced and were surgically re-closed on day 5 and again on day 7. Twelve days post-operatively his appetite decreased and 13 days post-operatively he was severely depressed, displayed nystagmus, and was euthanized.

In all 3 infected pigs, ventriculomegaly was seen at post-mortem CT, and during necropsy we observed purulent discharge centered around the valve and surgical site, and tracking down the ventricular catheter into the brain. In the two Treatment pigs, the purulent discharge obstructed the inlet pore. In the Control pig, there was also hematoma obstructing the lumen of the ventricular catheter. After two infections in the first seven surgeries, we administered two peri-operative doses of florfenicol to each animal and added a subcutaneous muscle suture layer. 
Ventricular volume The post-operative CT in our animals showed significantly larger ventricles than prior to surgery in both groups ( $p=0.002$, Fig. 5a). By week 1, all air had been resorbed. In the Treatment group, all hematoma had been resorbed and ventricular size had non-significantly decreased. In the Control group, there had been a further enlargement of the ventricles and in some animals hematoma persisted.

Figure $5 \mathrm{~b}$ shows the change in ventricular volume until 1 week post-implantation. Two-way ANOVA showed that statistical significance for both the device type and time. Specifically, the ventricular volume in the Control animals was significantly larger than in the Treatment group $(p<0.001)$. Moreover, the ventricular volume significantly increased before and after IVH and after a week of recovery $(p<0.001)$. Pairwise comparison showed that by week 1 , there was a significant difference in ventricular volume between the Control and the Treatment group $(p<0.001)$.

The data from W3 and W5 were omitted for statistical comparison because all Control shunts failed after week 1. The change in ventricle volume over the entire implantation period are shown in SI Fig. 6. Although the Treatment animals survived longer with our self-clearing catheters, the ventricle volume continued to increase, which may highlight the limitation of our study. This trend suggests that the self-clearing catheters are prolonging the functional lifetime but we may need to increase the actuation duty cycle to optimize treatment.

Kaplan-Meier survival analysis We performed Kaplan-Meier analysis to evaluate the survivability of the shunt system due to hematoma, censoring the death of pigs due to any other reason including anesthesia complication and infection. (Fig. 5c). The median shunt survival in the Treatment group was 42 days and the median shunt survival in the Control group was 3 days. This difference was statistically significant $(p=0.0047)$, which further supports our hypothesis that the self-clearing ventricular catheters are more reliable and capable than existing drainage devices. We repeated the Kaplan-Meier analysis considering infection as a shunt failure, but the difference still remained statistically significant and the $p$-value unchanged $(p=0.0047$, SI Fig. 7), which further supports our hypothesis that the self-clearing catheters may improve the outcomes of IVH and PHH patients.

\section{Discussion}

It is important to note that the prototype catheters we tested are at a much higher risk of failure than a standard ventricular catheter. Conventional ventricular catheters typically contain 16 or more inlet pores ( 3 to 4 rows of 4 to 8 inlet pores) ${ }^{53}$. The self-clearing catheter we used had a single inlet pore to accelerate the occlusion, even though it is possible to integrate an array into the catheter (SI Fig. 4c, SI Video 3). As such, the occlusion of this lone inlet pore would have led to a complete device failure, a circumstance very different from a conventional catheter. The single inlet pore also increased the chance of inadequate surgical placement: the rows of inlet pores in conventional catheters maximize the likelihood that multiple inlet pores will be located in the lateral ventricle.

In one Treatment pig, the post-operative CT indicated that this single inlet pore was too deep (passing through the ventricle and into brain tissue on the other side) and led to additional surgery and, most importantly, delayed actuation. This Treatment shunt was not actuated until after a second surgery to retract the ventricular catheter and was fatally obstructed in less than $24 \mathrm{~h}$. Upon explantation, we found that the hematoma had passed the microactuator within the tip of the ventricular catheter and was at the junction of the ventricular catheter and valve, suggesting the first actuation may not have occurred until after overwhelming hemorrhage had already passed the actuator. All other Treatment shunts were first actuated soon after placement and subsequently remained free of hematoma obstruction throughout the 6-week study.

There was an additional risk of device failure as the catheter was placed directly into the ventricular hematoma, through the tract used for injection of blood, being inserted immediately after the injection was completed. Since the blood was mixed with the coagulant agent thrombin, we suspect that a mixture of CSF, blood, and thrombin immediately entered the catheter to begin coagulating. In current clinical practice, the presence of hematoma is a contraindication for shunting, which is typically recommended only when hydrocephalus has been diagnosed ${ }^{7}$. We believe this highlights the novelty and the robustness of our approach that enables earlier usage of drainage system using our self-clearing catheters.

In addition, we used low opening pressure valves $(2.2 \mathrm{mmHg})$ as a part of the shunt system, which may have exacerbated the occlusion issue. The typically used valve in VP shunt surgery has a medium opening pressure $(6-7 \mathrm{mmHg})$, within the range for normal ventricular pressure of $5-15 \mathrm{mmHg}$. The low opening pressure would have increased the passage of hemorrhagic CSF through the VP shunt, as the coagulating fluid would have taken the path of least resistance rather than negotiate the ventricular system. Hemorrhage present within the ventricular system (e.g. foramen of Munro) would have been able to form a permanent thrombus, rather than being dislodged by CSF flow, causing the flow to be directed through the shunt system, which further highlights the potential utility of this approach.

It is also important to note that the injection of blood in the in-vivo experiment provides a substrate for bacterial growth. Neonates with PHH suffer from infection-related shunt failures in as many as $71 \%$ of cases ${ }^{54}$. Although our microactuators did not prevent failure due to infection, the use of antibiotic-impregnated catheters is associated with significantly fewer 
post-operative infections ${ }^{54}$. Thus, a logical next step may be to use a combination of microactuators with antibiotic or anti-inflammatory drug-eluting catheters to potentially overcome infection or immune-related device failures ${ }^{29}$.

We also compared the performance of our self-clearing catheter against the common clinical practice of using saline flush to remove drainage device obstruction. Although saline flushes are effective in removing the obstruction, each flushing caused a potentially dangerous level of pressure spike, and the catheter was rapidly re-occluded, which necessitated more frequent flushing. Pressure spikes notwithstanding, the flushed catheters remained above the safe pressure range for longer periods than our self-clearing catheters. Our results highlight the clinical challenge of having a person constantly monitoring patient ICP to ensure that the drainage device performs reliably until the hematoma clears. In comparison, our approach may be used with minimal user input and maintain patency better than existing devices by actuating the smart catheter using an optimized duty cycle.

Overall, our experimental results demonstrate a novel non-pharmaceutical approach of using magnetic microactuatorenabled smart catheters to treat IVH in situ using externally applied magnetic fields. We demonstrated that these magnetic microactuators can expedite the removal of blood from the ventricle, maintain a lower ventricle volume, and increase the survival rate of IVH-suffering animal. Although additional work is needed, we believe these types of magnetic microactuator-embedded smart catheters can potentially improve the lifetime and the reliability of chronically implanted catheters that are critical in a number of neurological, urological, cardiovascular, and other drug delivery applications.

\section{Methods}

\section{Device fabrication}

The microactuators were fabricated in a standard cleanroom environment. Starting from a 100 mm p-type single-side polished silicon wafer (Silicon Quest, San Jose, CA), $50 \mathrm{~nm}$ of silicon dioxide was deposited by plasma-enhanced chemical vapor deposition (Axic, Milpitas, CA) to function as a release layer. Next, a layer of polyimide PI-2525, HD Microsystem, Parlin, $\mathrm{NJ}$ ) was spun coated at $1750 \mathrm{rpm}$ and cured in a nitrogen oven up to $350{ }^{\circ} \mathrm{C}$ to create a final thickness of $11 \mu \mathrm{m}$. A 50 -nmthick Cr was evaporated (Airco E-Beam Evaporator, Livermore, CA) as the etch mask for the polymer layer. Cr mask was photo-patterned using AZ9260 (Microchemicals, Germany) and etched using a Cr etchant (Cr-16, KMG, Fort Worth, TX). The polyimide structural layer was etched using $\mathrm{O}_{2}$ plasma at $20 \mathrm{sccm}, 50 \mathrm{mTorr}$ and $150 \mathrm{~W}$ RF power (Advanced Oxide Etcher, Surface Technology System, Newport, UK). Cr mask was then removed using Cr-16.

Next, $100 \mathrm{~nm}$ of Au was sputtered (PVD, Wilmington, MA) globally as a conduction layer and 200- $\mu$ m-thick electroplating mold was photo-patterned using a negative photoresist (BPN-65A, Rohm Haas, Marlborough, MA). The nickel ferromagnetic elements were in $2 \mathrm{~L}$ plating solution maintained at $60{ }^{\circ} \mathrm{C}$ with $40 \mathrm{~mA}$ direct current for $4 \mathrm{~h}$. The plating solution contained $1 \mathrm{M}$ nickel sulfamate, $0.4 \mathrm{M}$ boric acid and $10 \mathrm{~g}$ sodium dodecyl sulfate. The electroplated elements varied from $80-130 \mu \mathrm{m}$ in thickness depending on the actuator geometry. Afterward, the exposed Au conduction layer was stripped using a wet etchant (GE8148, Transene, Danvers, MA). The microactuators were released from the wafer using a buffered oxide etchant (Fisher Scientific, Waltham, MA). To create the self-clearing catheter, the released sample was rolled and inserted into a catheter lumen (Model G0664, Cook Medical) as previously described ${ }^{25}$.

\section{Finite element analysis}

COMSOL Multiphysics (V5.0, COMSOL, Inc., Burlington, MA, USA) was used for the finite element analysis of device deflection and stress distribution. Each device design was configured to have the material property of polyimide with a density of $1300 \mathrm{~kg} / \mathrm{m}^{3}$, Young's modulus of $2.45 \mathrm{GPa}$ and poison ratio of 0.3 . Using the solid mechanics module, each flexure was fixed on one end and a vertical point load ranging from 1-100 $\mu \mathrm{N}$ was applied to the free end to evaluate the static deflection and the stress distribution. Lagrange strain was used to account for the large flexure deformation.

\section{Static response}

The microactuators were positioned along the long axis of a bespoke solenoid electromagnet (cylindrical permalloy core, 1-in-diameter and 6-in-tall with 300 turns) and down to the edge of a glass slide. The distance between the sample and the electromagnet surface was kept at $7 \mathrm{~mm}$ to minimize the magnetic flux density gradient $(<0.1 \mathrm{~T} / \mathrm{m})$ while maintaining an adequate amount of parallel magnetic field strength. A DC power source (PWS2326, Tektronix, Beaverton, Oregon) was used to supply the current to the electromagnet. The amount of magnetic flux density at the position of actuators was measured using gaussmeter (8010, F.W. Bell, Milwaukie, OR). During actuation, the electromagnet along with actuator positioning glass slides were placed horizontally and the amount of deflection angle was optically measured using a digital SLR camera (Canon 50D, Huntington, NY). The magnetic flux density was varied from $0-22 \mathrm{mT}$ for the straight beamed device and from $0 \mathrm{mT}$ to $17 \mathrm{mT}$ for the serpentine device. 


\section{In vitro evaluation}

A $50 \mathrm{ml}$ glass bottle (Duran, Fisher Scientific, Waltham, MA) was used to mimic the ventricular chamber. The bottle was sealed using a screw cap with two through holes allowing placement of the inlet and outlet tubing (L/S 14, Masterflex, Cole-Parmer). A piece of a central venous catheter (Model G0664, Cook Medical) was attached to the outlet inside the glass bottle chamber. The through-holes on the screw cap was sealed using a silicone adhesive to fix the tubings in place. For the catheters used for flushing, a three-way stopcock (Masterflex, Fisher Scientific, Waltham, MA) was introduced to the outlet of the ventricle chamber. A $10 \mathrm{~mL}$ syringe (Model G0664, Cook Medical) with $2 \mathrm{~mL}$ of 1X PBS (Fisher Scientific, Waltham, MA) was attached to one of the inlets of the valve. A variable speed peristaltic pump (7523-50, Masterflex, Cole Parmer) was used to drive fluid into the chamber at $1.4 \mathrm{ml} / \mathrm{min}$, about twice the rate of average cerebral spinal fluid production in humans ${ }^{55,56}$. Two pressure sensors (PRESS-S-00, PendoTech, Princeton, NJ) were connected to the inlet and the outlet tubes.

A mixture of porcine blood and 1X PBS (Fisher Scientific, Waltham, MA) at 50:50 vol was used to mimic hemorrhagic cerebrospinal fluid. The fresh blood of euthanized pigs was mixed with 10 USP units of heparin/ml and stored in a refrigerator at $4{ }^{\circ} \mathrm{C}$ for 1 week prior to the experiment. At the time of testing, protamine sulfate (Fisher Scientific, Waltham, MA) was added (10 mg per 100 USP heparin) to reverse the effects of anti-coagulant and to facilitate blood clot formation ${ }^{57}$. Since conventional catheter occlusion is unpredictable with a wide range of time to failure ${ }^{58,59}$, a layer of fibrin matrix gel was applied on the catheter surface surrounding the inlet pore to further promote blood clots attachment. The fibrin gel was made from a mixture of fibrinogen $(38 \mathrm{mg} / \mathrm{ml})$ and thrombin $(37 \mathrm{mg} / \mathrm{ml})$ (Thermo Fisher Scientific, Waltham, MA) ${ }^{60-62}$.

Each device was subjected to $4 \mathrm{~h}$ of circulation in the circulating setup during which the differential pressure was recorded continuously (12 samples/min $)^{24}$. For Control $(n=4)$ and Flush $(n=6)$ groups, single-pore catheters without any microactuators were tested. For Treatment groups, self-clearing catheters with either straight $(n=5)$ and serpentine flexure designs were tested $(n=6)$. During each experiment, the time-varying magnetic field was applied using a permanent magnet (McMaster-Carr, Elmhurst, IL) affixed to a DC motor from $20 \mathrm{~mm}$ away spinning at $8 \mathrm{~Hz}$. The magnetic performance of this motorized setup is shown in SI Fig. 13. Using the differential pressure recording, the time to reach a threshold pressure (i.e., time to occlusion, TTO) and the time over a threshold pressure (TOT, $40 \mathrm{mmHg}$ ) was calculated. The TTO and TOT for different conditions were compared using one-way ANOVA with Tukey's HSD post-hoc analyses with $p<0.05$ as statistical significance.

The ultrastructure of hematoma fragments was analyzed for the integrity of the fibrin fiber network. All blood-PBS solution exiting the $\mathrm{VC}$ was collected directly into a $200 \mathrm{~mL} 4 \%$ glutaraldehyde and $4 \%$ paraformaldehyde fixative for preparation for SEM hematoma fragment analysis. The blood-PBS fixed solution was prepared for SEM analysis using previously described methods ${ }^{63}$. Samples were washed with three cycles of PBS (5 min each). Secondary fixation was performed with $1 \%$ osmium tetroxide for $1 \mathrm{~h}$. The fragments were rinsed in de-ionized water three times at $5 \mathrm{~min}$ per cycle. All samples were dehydrated in a series of ethanol solutions of increasing concentration for 10 min each $(10 \%, 30 \%, 50 \%, 70 \%, 85 \%$, two times at $95 \%$ and three times at 100\%) followed by critical point drying. Finally, samples were placed on aluminum mounts and carbon-coated prior to SEM. A grading scale was developed to semi-quantitatively measure the samples (SI Fig. 5). Samples were analyzed and scored by a board-certified (American College of Veterinary Pathologists) clinical pathologist. All control $(n=36)$ and treatment $(n=18)$ samples were analyzed and scored by a board-certified (American College of Veterinary Pathologists) clinical pathologist.

\section{In vivo evaluation}

For the in vivo evaluation, 13 cross-bred domestic swines weighing 25.0-31.6 kg were used (Fig. 4). Each pig was pre-medicated with $0.2 \mathrm{mg} / \mathrm{kg}$ midazolam (Hikma Pharmaceuticals USA Inc, Eatontown, NJ) and $0.03 \mathrm{mg} / \mathrm{kg}$ dexmedetomidine (Zoetis US, Parsippany, NJ, USA) and induced with isoflurane delivered by face mask until tracheal intubation was possible. Anesthesia was continued with isoflurane delivered in $100 \%$ oxygen. Mechanical ventilation, pulse oximetry, capnography, indirect blood pressure measurement, temperature measurement, left jugular central line placement and intravenous fluid therapy (10 $\mathrm{mL} / \mathrm{kg} / \mathrm{hr}$ ) were performed. Analgesia was provided by the pre-operative transdermal application of $2.7 \mathrm{mg} / \mathrm{kg}$ fentanyl solution (4-day expected activity) (Elanco Geenfield, IN). Intramuscular injection of $5 \mathrm{mg} / \mathrm{Kg}$ ceftiofur (7-day expected activity) was used for antibiotic treatment.

To induce intraventricular hemorrhage, a right side subtemporal craniectomy was performed using standard aseptic technique. To measure intraventricular ICP, a 1.25-inch 18-gauge intravenous cannula was directed through intact dura mater into the right lateral ventricle. The correct ventricular location was confirmed by drainage of CSF. Non-compliant tubing (pre-filled with saline) was connected tightly and attached to a transducer and monitor. To inject the blood-thrombin mixture, a second 1.25-inch 18-gauge intravenous cannula was then placed into the lateral ventricle, more rostrally. The drainage of CSF again confirmed accurate placement. A total of $10 \mathrm{ml}$ of autologous blood and 140 units thrombin was injected through this cannula as determined using preliminary studies. Blood was drawn from the jugular catheter using sterile technique in 3 equal aliquots. Each aliquot was briefly agitated with thrombin in a syringe and then injected. Each time ICP rose above $50 \mathrm{mmHg}$, the injection was paused until ICP was $<50 \mathrm{mmHg}$. This second cannula was then withdrawn. 
The puncture hole in the dura mater was slightly enlarged with a \#11 scalpel blade, and the ventricular catheter was inserted through the tract that had been used for blood injection. In 6 pigs, a Control VP shunt was placed. The ventricular catheter was custom-made for this study using a modified central venous access device (Cook Model G0664, Bloomington, IN), with only one single inlet pore. In 7 pigs, a Treatment shunt was placed. The custom-made ventricular catheter had a microactuator placed at the single inlet pore ${ }^{25}$. The correct ventricular location was confirmed by rapid drainage of sanguineous CSF. ICP recording was continued until 30 min after the placement of the ventricular catheter.

The ventricular catheter was anchored to the skull by a pre-placed polydioxanone suture (Ethicon, Somerville, NJ) through the periosteum and loose fascia. It was connected to a pre-placed low-pressure valve (Integra Lifesciences, Plainsboro, NJ) and distal catheter which had been filled with saline, creating a VP shunt. The valve was anchored to the periosteal tissue of the posterior skull by a suture that encircled the connection of the ventricular catheter to the valve. The valve was pumped, and the functionality of the VP shunt was confirmed by sanguineous CSF entering the valve and saline escaping from the distal catheter. The distal catheter was placed in the peritoneal cavity and the paracostal incision closed. The fascia of the temporalis muscle was closed with a simple continuous polydioxanone suture. The skin was closed with simple continuous subcuticular and intradermal poliglecaprone sutures (Ethicon), followed by the application of skin glue.

Computed tomography was performed prior to and immediately following surgery (day 0 ). In the Treatment group, actuation was performed immediately after post-operative CT for 30 minutes using a bespoke electromagnet (SI Fig. 14). The electromagnet has a maximum magnetic field strength of $50 \mathrm{kA} / \mathrm{m}$ which is orders of magnitude below the necessary magnetic field strength for transcranial magnetic stimulation ${ }^{64}$. Pigs were then recovered from anesthesia. Repeat CT was performed at weeks 1,3 , and 5. Pigs were again premedicated with $0.2 \mathrm{mg} / \mathrm{kg}$ midazolam and $0.03 \mathrm{mg} / \mathrm{kg}$ dexmedetomidine, then had oxygen and isoflurane delivered by face mask throughout the CT. In the Treatment group, repeat actuation was performed immediately after each CT. The first CT was 5 to 7 days post-operatively so that the first actuation was performed at day 5 or 6 in the Treatment group.

The size of the ventricles was measured using a DICOM viewer (Osirix Lite 10.0.1, Pixmeo SARL Bernex, Switzerland). The ventricle (i.e., region of interest) from each CT scan was outlined manually by three measurers. The volume of the outlined region of interest was calculated by the DICOM viewer and compared (SI Fig. 15). One-way ANOVA with Tukey's HSD post-hoc analysis showed no statistical significance between the three measurers $(p=0.095)$. To compare the difference in ventricle volumes in the Treatment vs. the Control group, two-way ANOVA was performed to see the effects of time and the type of catheters. A post-hoc pairwise comparisons were made using Tukey's HSD test.

Kaplan-Meier analysis of shunt survival was used to compare Treatment and Control groups. Obstruction of the shunt by hematoma was considered an event. Both for fatal shunt obstructions and non-fatal shunt obstructions causing neurological decline, we noted the increased size of the lateral ventricles on CT and we verified the shunt obstruction by hematoma at necropsy when these animals were sacrificed at day 42. Death of the pig due to any other reasons (e.g., anesthesia complication, infection) was censored.

\section{References}

1. Steiner, T. et al. Dynamics of intraventricular hemorrhage in patients with spontaneous intracerebral hemorrhage: Risk factors, clinical impact, and effect of hemostatic therapy with recombinant activated factor VII. Neurosurgery 59, 767-773 (2006).

2. Qureshi, A. I., Mendelow, A. D. \& Hanley, D. F. Intracerebral haemorrhage. The Lancet 373, 1632-1644 (2009).

3. Murphy, B. P. et al. Posthaemorrhagic ventricular dilatation in the premature infant: natural history and predictors of outcome. Archives of Disease in Childhood. Fetal and Neonatal Edition 87, F37-41 (2002).

4. Guzzetta, F., Mercuri, E. \& Spanò, M. Mechanisms and evolution of the brain damage in neonatal post-hemorrhagic hydrocephalus. Child's Nervous System 11, 293-296 (1995).

5. Gaberel, T. et al. Urokinase versus Alteplase for intraventricular hemorrhage fibrinolysis. Neuropharmacology 85, 158-165 (2014).

6. Basaldella, L. et al. External ventricular drainage alone versus endoscopic surgery for severe intraventricular hemorrhage: a comparative retrospective analysis on outcome and shunt dependency. Neurosurgical Focus 32, E4 (2012).

7. Hemphill, J. C. et al. Guidelines for the Management of Spontaneous Intracerebral Hemorrhage: A Guideline for Healthcare Professionals from the American Heart Association/American Stroke Association. Stroke 46, 2032-2060 (2015).

8. Mwachaka, P. M., Obonyo, N. G., Mutiso, B. K., Ranketi, S. \& Mwang’Ombe, N. Ventriculoperitoneal shunt complications: A three-year retrospective study in a Kenyan national teaching and referral hospital. Pediatric Neurosurgery 46, 1-5 (2010).

9. Tanrıkulu, B. \& Özek, M. M. Mechanical Shunt Complications, 1-23 (Springer International Publishing, Cham, 2018). 
10. Naff, N. J. et al. Intraventricular thrombolysis speeds blood clot resolution: Results of a pilot, prospective, randomized, double-blind, controlled trial. Neurosurgery 54, 577-584 (2004).

11. Goldstein, J. N. \& Greenberg, S. M. Should anticoagulation be resumed after intracerebral hemorrhage? Cleveland Clinic Journal of Medicine 77, 791-799 (2010).

12. van Solinge, T. S. et al. Fibrinolytics and Intraventricular Hemorrhage: A Systematic Review and Meta-analysis. Neurocritical Care 32, 262-271 (2020).

13. Sainte-Rose, C. et al. Mechanical Complications in Shunts. Pediatric Neurosurgery 17, 2-9 (1991).

14. Kocan, M. J. Ask the Experts. Critical Care Nurse 22, 70-73 (2002).

15. Adams, R. E. \& Diringer, M. N. Response to external ventricular drainage in spontaneous intracerebral hemorrhage with hydrocephalus. Neurology 50, 519-523 (1998).

16. Hanley, D. F., Fisher, M. \& Lees, K. Intraventricular hemorrhage severity factor and treatment target in spontaneous intracerebral hemorrhage. Stroke 40, 1533-1538 (2009).

17. Morales, D. M. et al. Cerebrospinal fluid levels of amyloid precursor protein are associated with ventricular size in post-hemorrhagic hydrocephalus of prematurity. PLOS ONE 10, 1-14 (2015).

18. Staykov, D. et al. Single versus bilateral external ventricular drainage for intraventricular fibrinolysis in severe ventricular haemorrhage. Journal of Neurology, Neurosurgery, \& Psychiatry 81, 105 LP - 108 (2010).

19. Hinson, H. E. et al. Drainage Efficiency with Dual Versus Single Catheters in Severe Intraventricular Hemorrhage. Neurocritical Care 16, 399-405 (2012).

20. Rosenschein, U. et al. Ultrasound imaging-guided noninvasive ultrasound thrombolysis: Preclinical results. Circulation 102, 238-245 (2000).

21. Nedelmann, M. et al. Ultrasound-Induced Blood Clot Dissolution without a Thrombolytic Drug Is More Effective with Lower Frequencies. Cerebrovascular Diseases 20, 18-22 (2005).

22. Kucher, N. et al. Randomized controlled trial of ultrasound-assisted catheter-directed thrombolysis for acute intermediaterisk pulmonary embolism. Circulation 129, 479-486 (2013).

23. Kim, J. et al. Intravascular forward-looking ultrasound transducers for microbubble-mediated sonothrombolysis. Scientific Reports 7, 1-10 (2017).

24. Lee, H., Kolahi, K., Bergsneider, M. \& Judy, J. W. Mechanical evaluation of unobstructing magnetic microactuators for implantable ventricular catheters. Journal of Microelectromechanical Systems 23, 795-802 (2014).

25. Yang, Q. et al. Anti-biofouling implantable catheter using thin-film magnetic microactuators. Sensors and Actuators B: Chemical 273, 1694-1704 (2018).

26. Park, H., Raffiee, A. H., John, S. W. M., Ardekani, A. M. \& Lee, H. Towards Smart Self-Clearing Glaucoma Drainage Device. Microsystems \& Nanoengineering 4 (2018).

27. Erkoc, P. et al. Mobile Microrobots for Active Therapeutic Delivery. Advanced Therapeutics 2, 1800064 (2019).

28. Huang, H. W., Tibbitt, M. W., Huang, T. Y. \& Nelson, B. J. Matryoshka-Inspired Micro-Origami Capsules to Enhance Loading, Encapsulation, and Transport of Drugs. Soft Robotics 6, 150-159 (2019).

29. Yasa, I. C., Ceylan, H., Bozuyuk, U., Wild, A. M. \& Sitti, M. Elucidating the interaction dynamics between microswimmer body and immune system for medical microrobots. Science Robotics 5, 1-14 (2020).

30. Tang, S. et al. Enzyme-powered Janus platelet cell robots for active and targeted drug delivery. Science Robotics 5, 41-43 (2020).

31. Jeon, S. et al. Magnetically actuated microrobots as a platform for stem cell transplantation. Science Robotics 4, 1-12 (2019).

32. Kim, Y., Parada, G. A., Liu, S. \& Zhao, X. Ferromagnetic soft continuum robots. Science Robotics 4, 1-16 (2019).

33. Li, C. et al. Fast and programmable locomotion of hydrogel-metal hybrids under light and magnetic fields. Science Robotics 5 (2020).

34. Yang, X. et al. An agglutinate magnetic spray transforms inanimate objects into millirobots for biomedical applications. Science robotics 5, 1-13 (2020).

35. Li, J., Ávila, B. E. F. D., Gao, W., Zhang, L. \& Wang, J. Micro/nanorobots for Biomedicine: Delivery, surgery, sensing, and detoxification. Science Robotics 2, 1-10 (2017). 
36. Yang, Q., Lee, A., Bentley, R. T. \& Lee, H. Piezoresistor-embedded multifunctional magnetic microactuators for implantable self-clearing catheter. IEEE Sensors Journal 19, 1373-1378 (2019).

37. Karami, M. A. \& Inman, D. J. Analytical modeling and experimental verification of the vibrations of the zigzag microstructure for energy harvesting. Journal of Vibration and Acoustics 133, 11002 (2011).

38. Berdy, D. F. et al. Low-frequency meandering piezoelectric vibration energy harvester. IEEE Transactions on Ultrasonics, Ferroelectrics, and Frequency Control 59, 846-858 (2012).

39. Amin Karami, M. \& Inman, D. J. Powering pacemakers from heartbeat vibrations using linear and nonlinear energy harvesters. Applied Physics Letters 100, 42901 (2012).

40. Karami, M. A. \& Inman, D. J. Electromechanical modeling of the low-frequency zigzag micro-energy harvester. Journal of Intelligent Material Systems and Structures 22, 271-282 (2011).

41. Lee, H., Xu, Q., Shellock, F. G., Bergsneider, M. \& Judy, J. W. Evaluation of magnetic resonance imaging issues for implantable microfabricated magnetic actuators. Biomedical Microdevices 16, 153-161 (2014).

42. Nelson, B. J., Kaliakatsos, I. K. \& Abbott, J. J. Microrobots for minimally invasive medicine. Annual Review of Biomedical Engineering 12, 55-85 (2010).

43. Andrä, W. \& Nowak, H. Magnetism in Medicine: A Handbook (John Wiley \& Sons, 2007).

44. Nishimura, D. G. Principles of Magnetic Resonance Imaging (Standford Univ., 2010).

45. Lee, S. A. et al. Development of microfabricated magnetic actuators for removing cellular occlusion. Journal of Micromechanics and Microengineering 21, 54006 (2011).

46. Czosnyka, Z., Czosnyka, M., Richards, H. K. \& Pickard, J. D. Posture-related overdrainage: comparison of the performance of 10 hydrocephalus shunts in vitro. Neurosurgery 42, 327-334 (1998).

47. Bratton, S. L. et al. Guidelines for the management of severe traumatic brain injury. VIII. Intracranial pressure thresholds. Journal of Neurotrauma 24, S55-8 (2007).

48. Chambers, I. R. et al. Critical thresholds of intracranial pressure and cerebral perfusion pressure related to age in paediatric head injury. Journal of Neurology, Neurosurgery \& Psychiatry 77, 234-240 (2006).

49. Smith, M. Monitoring intracranial pressure in traumatic brain injury. Anesthesia \& Analgesia 106, 240-248 (2008).

50. Yamane, T. et al. The most profitable use of flow visualization in the elimination of thrombus from a monopivot magnetic suspension blood pump. Artificial Organs 28, 390-397 (2004).

51. Hashimoto, S., Maeda, H. \& Sasada, T. Effect of shear rate on clot growth at foreign surfaces. Artificial Organs 9, 345-350 (1985).

52. Runyon, M. K., Kastrup, C. J., Johnson-Kerner, B. L., Van Ha, T. G. \& Ismagilov, R. F. Effects of shear rate on propagation of blood clotting determined using microfluidics and numerical simulations. Journal of the American Chemical Society 130, 3458-3464 (2008).

53. Kestle, J. R. W. CSF shunts 50 years on past, present and future. Child's Nervous System 16, 800-804 (2000).

54. Raffa, G., Marseglia, L., Gitto, E. \& Germanò, A. Antibiotic-impregnated catheters reduce ventriculoperitoneal shunt infection rate in high-risk newborns and infants. Child's Nervous System 31, 1129-1138 (2015).

55. Lutz, B. R., Venkataraman, P. \& Browd, S. R. New and improved ways to treat hydrocephalus: Pursuit of a smart shunt. Surgical Neurology International 4, S38-50 (2013).

56. Gideon, P. et al. Cerebrospinal fluid flow and production in patients with normal pressure hydrocephalus studied by MRI. Neuroradiology 36, 210-215 (1994).

57. Jaques, L. B. Protamine-antagonist to heparin. Canadian Medical Association Journal 108, 1291 (1973).

58. McGirt, M. J. et al. Cerebrospinal fluid shunt survival and etiology of failures: a seven-year institutional experience. Pediatric Neurosurgery 36, 248-255 (2002).

59. Kahle, K. T., Kulkarni, A. V., Limbrick Jr, D. D. \& Warf, B. C. Hydrocephalus in children. The Lancet 387, 788-799 (2016).

60. Mustard, J. F. \& Packham, M. A. Factors influencing platelet function: adhesion, release, and aggregation. Pharmacological Reviews 22, 97-187 (1970).

61. Guthold, M. et al. A comparison of the mechanical and structural properties of fibrin fibers with other protein fibers. Cell Biochemistry and Biophysics 49, 165-181 (2007). 
62. Duong, H., Wu, B. \& Tawil, B. Modulation of 3D fibrin matrix stiffness by intrinsic fibrinogen-thrombin compositions and by extrinsic cellular activity. Tissue Engineering Part A 15, 1865-1876 (2009).

63. Pretorius, E., Swanepoel, A. C., DeVilliers, S. \& Bester, J. Blood clot parameters: Thromboelastography and scanning electron microscopy in research and clinical practice. Thrombosis Research 154, 59-63 (2017).

64. Hallett, M. Transcranial Magnetic Stimulation: A Primer. Neuron 55, 187-199 (2007).

\section{Acknowledgements}

This work was supported NIH NINDS (R21NS095287), NIH/NCRR Indiana CTSI (UL1TR001108, UL/ TR002529), and Clinical and Translational Science Award and The Advances in Medicine (AIM) grant from Cook Medical. This work was also supported in part by the National Science Foundation (United States) under grants ECCS-1944480. The authors would like to thank Dr. Tiffany Lyle for her help in pathological analysis, Dr. George E. Moore for his help with the survival analysis, and Dr. Bradley Bohnstedt from the Indiana University School of Medicine for his critical discussion on the clinical utility of our approach. 


\section{Figures}
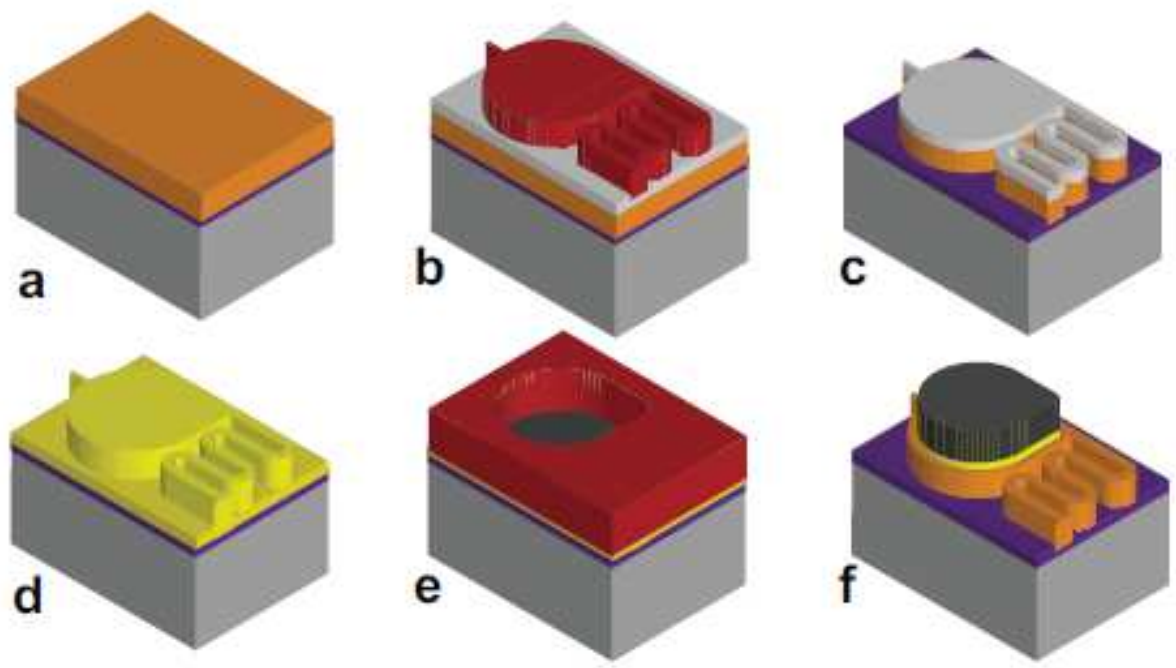

$\mathrm{Si} \quad \mathrm{SiO}_{2}$

Photoresist

\section{Polyimide}

$\mathrm{Cr} \square \mathrm{Ni}$

$\mathrm{Au}$

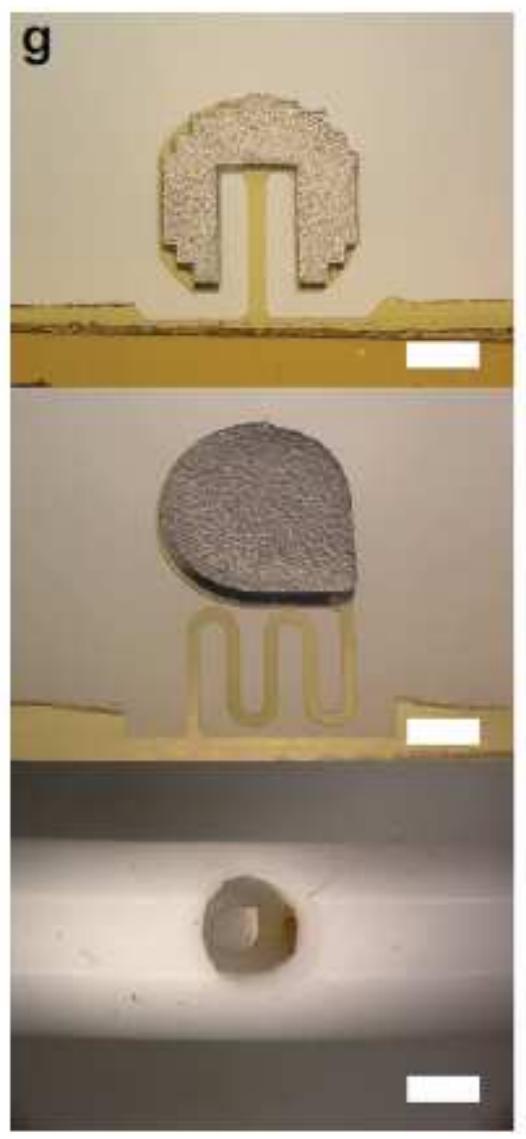

h
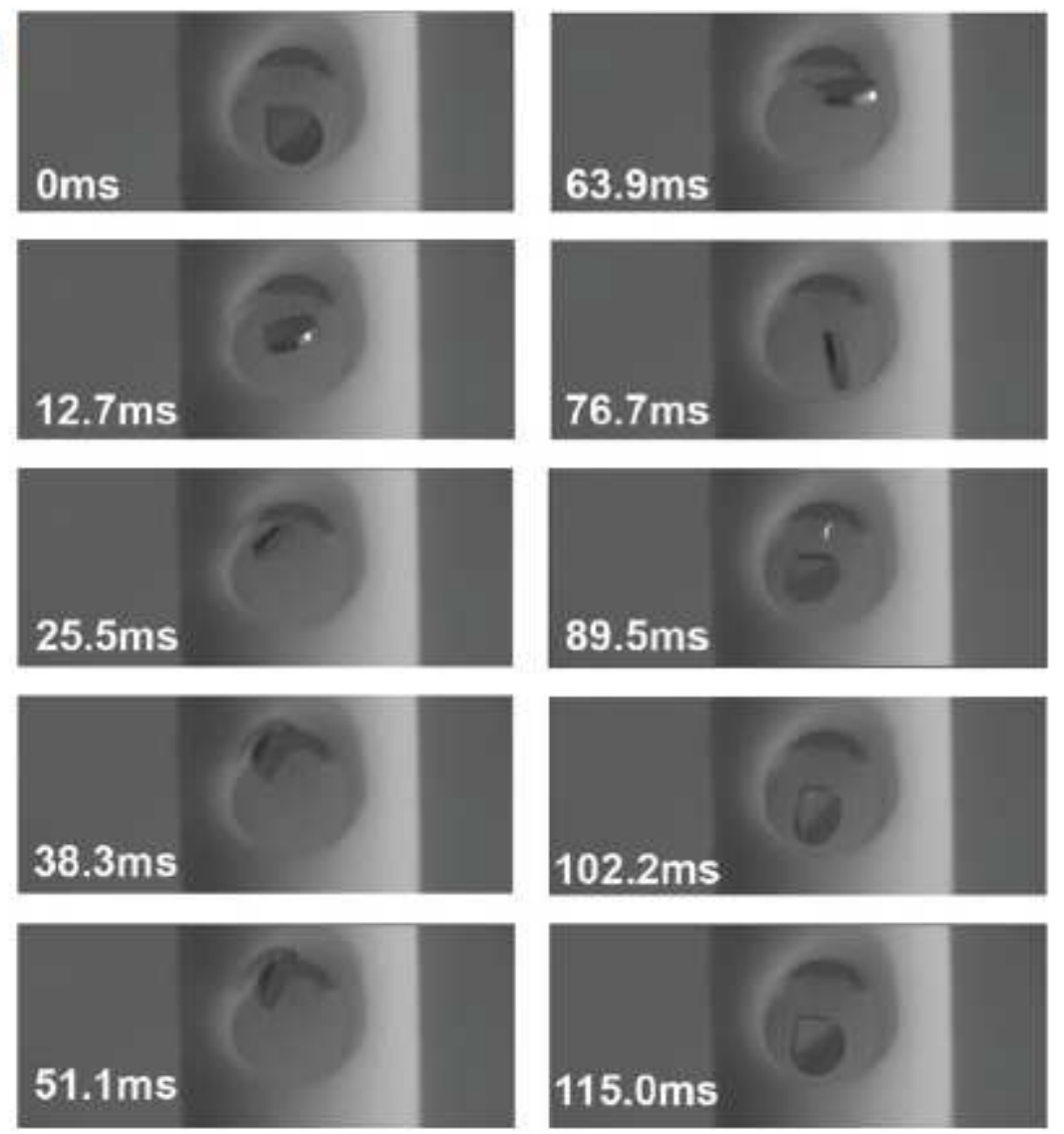

\section{Figure 1}

Design and fabrication of a self-clearing ventricular catheter. (a) Polyimide spin coat and curing. (b) Evaporation of $\mathrm{Cr}$ etching mask and define photoresist for actuator outline. (c) Wet etch of $\mathrm{Cr}$ and dry etch of polyimide. (d) Removal of $\mathrm{Cr}$ and evaporation of $\mathrm{Au}$ as a conduction layer. (e) application of photoresist and Ni electroplating. (f) removal of photoresist and remaining Au layer. (g) Optical images of fabricated magnetic actuators of straight type (top) and serpentine type (middle). Scale bars $=325 \mathrm{~mm}$. A 
catheter with an integrated actuator (bottom). Scale bar $=1 \mathrm{~mm}$. (h) A typical motion of actuation in deionized (DI) water for one actuation period.

a

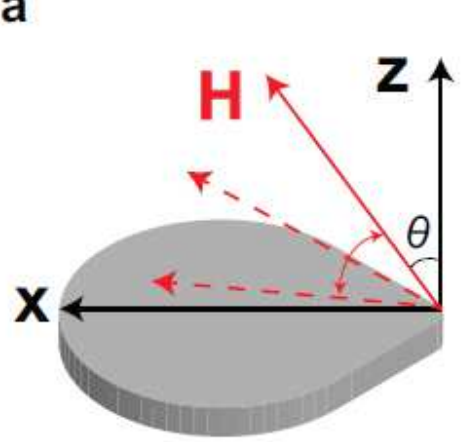

d

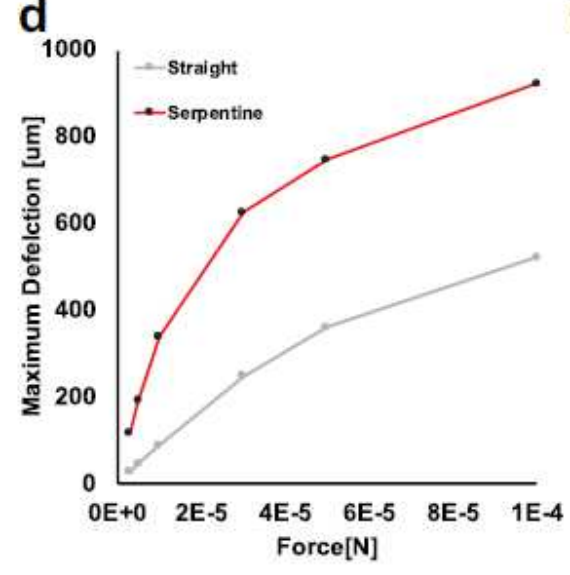

b

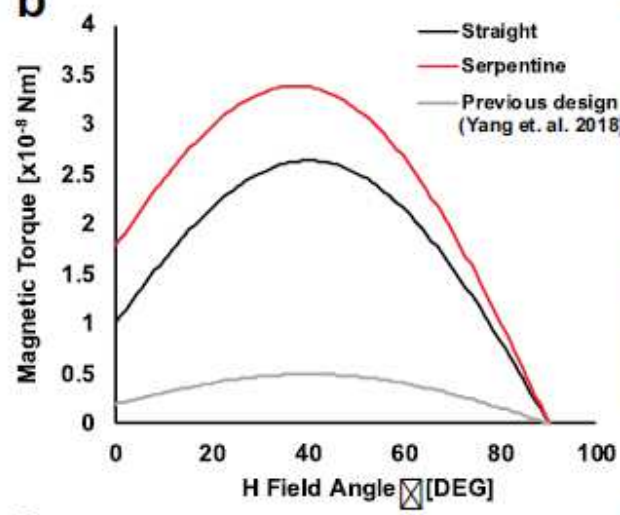

e

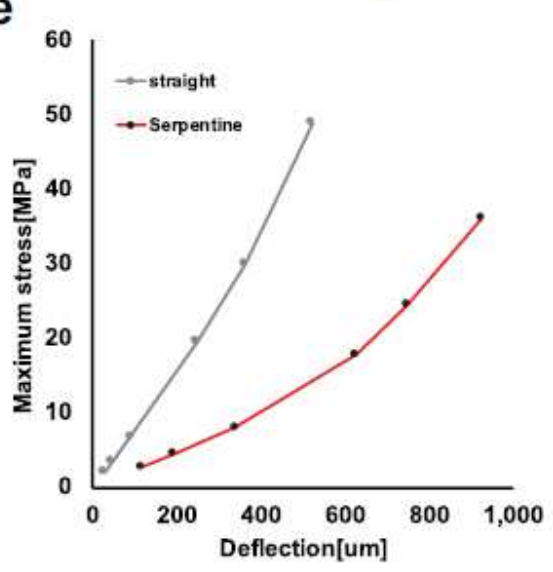

C

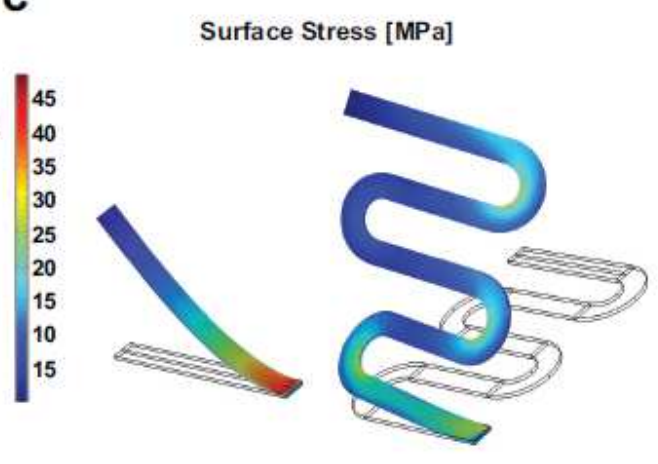

f

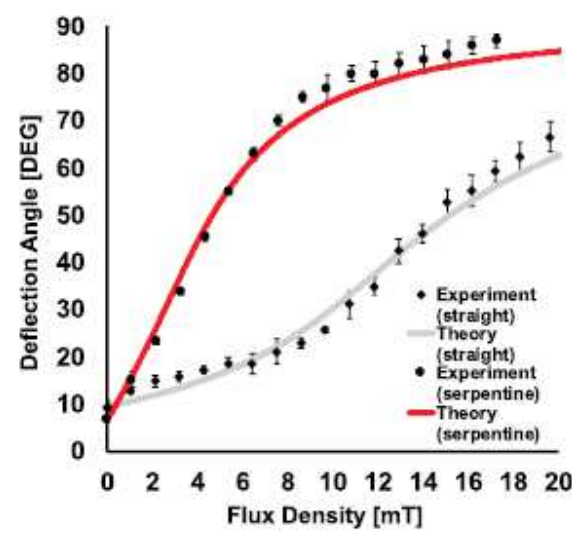

Figure 2

Mechanical characterization. (a) Definition of the coordinate system and magnetic field angle q. (b) Calculated magnetic torque produced on the ferromagnetic element of serpentine (red line) and straight actuator (black line) under $15 \mathrm{mT}$ at different field angles. (c) Finite element analysis of stress distribution on cantilevers' surfaces under $0.1 \mathrm{mN}$ load at the tip. (d) Maximum calculated deflection under loading condition ( 0.01 to $0.1 \mathrm{mN}$ ) for both cantilever type from finite element simulation. (e) Maximum calculated stress under various deflection conditions for both cantilever type from finite element simulation. (f) Static deflection angle prediction and measurements for the serpentine and the straight flexures. Data are expressed as mean \pm standard deviation $(n=3)$. 
a
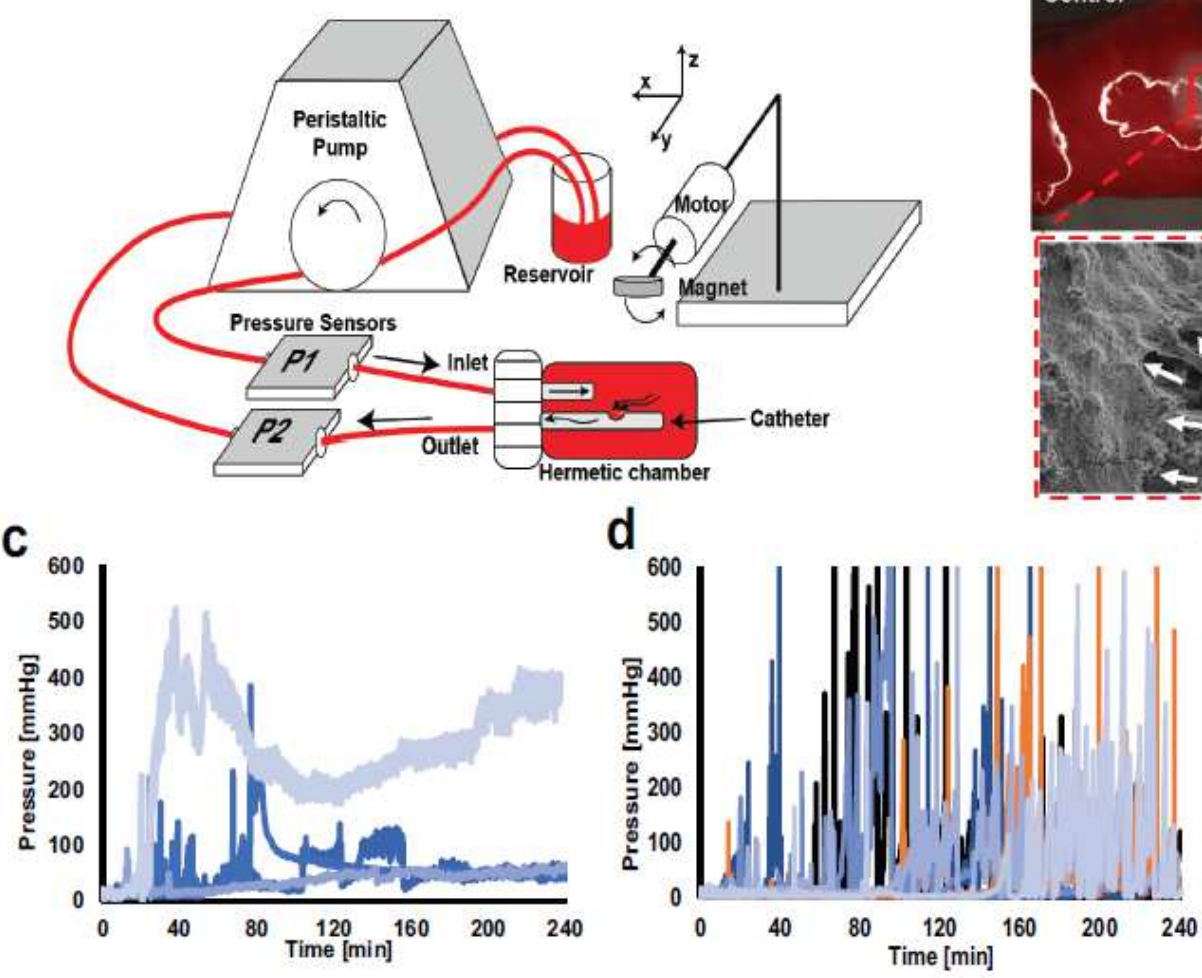

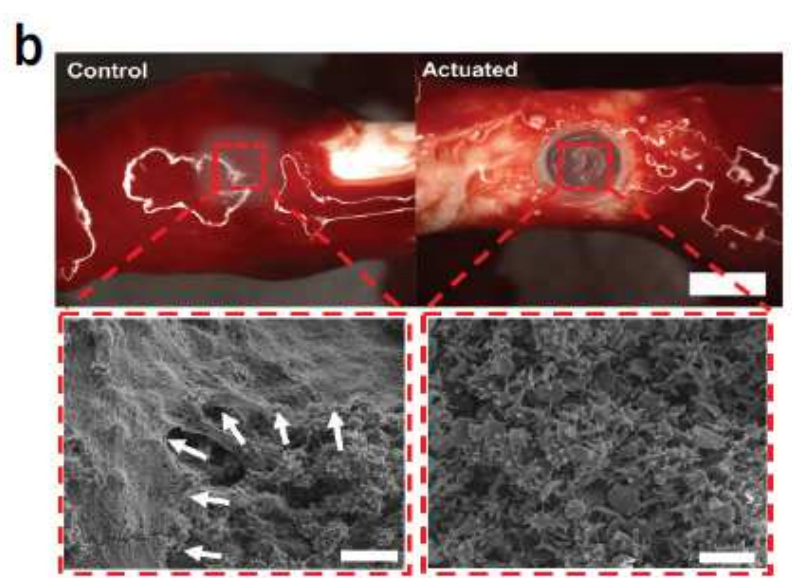

e
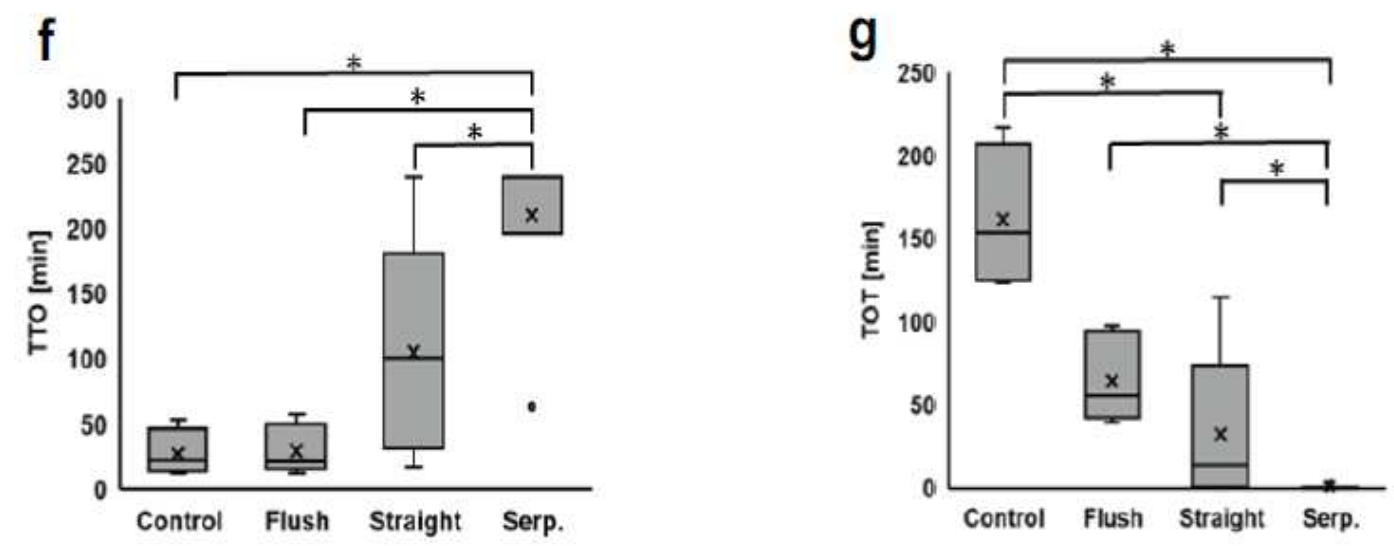

\section{Figure 3}

In vitro experimental setup and results. (a) Schematic of the bench-top blood circulation and magnetic actuation setup. (b) A representative visual comparison of control vs. actuated self-clearing catheter at the conclusion of the experiment. Scale bar $=1.5 \mathrm{~mm}$. Inset: SEM images obtained from hematoma fragments of control group. White arrows depict the robust fibrin network left unscathed and covering red blood cells. The hematoma fragments of the actuating group lack the fibrin network surrounding the red blood cells. Scale bar $=10 \mathrm{~mm}$. (c) Differential pressure (P2-P1) recording of all control catheters $(n=4)$. (d) Differential pressure (P2-P1) recording of all flushing catheters $(n=6)$. Note the frequent pressure spikes associated with each flushing. (e) Differential pressure (P2-P1) recording of all self-clearing catheters $(n=11)$. The figure includes data from 5 straight and 6 serpentine devices. (f) Comparison of time-to-occlusion (TTO) to reach $40 \mathrm{mmHg}$ between the control catheter, catheters that underwent flushing, and self-clearing catheters. There were two versions of self-clearing catheters with either straight 
vs. serpentine flexure microactuators. One-way ANOVA with Tukey post-hoc analysis showed overall statistical significance between groups. Individual comparison and significance $(p<0: 01)$ are indicated with *. (g) Comparison of the total time over the threshold (TOT). One-way ANOVA with Tukey post-hoc analysis confirmed statistical significance between groups. Individual comparison and significance $(p<$ 0:01) are indicated with *.

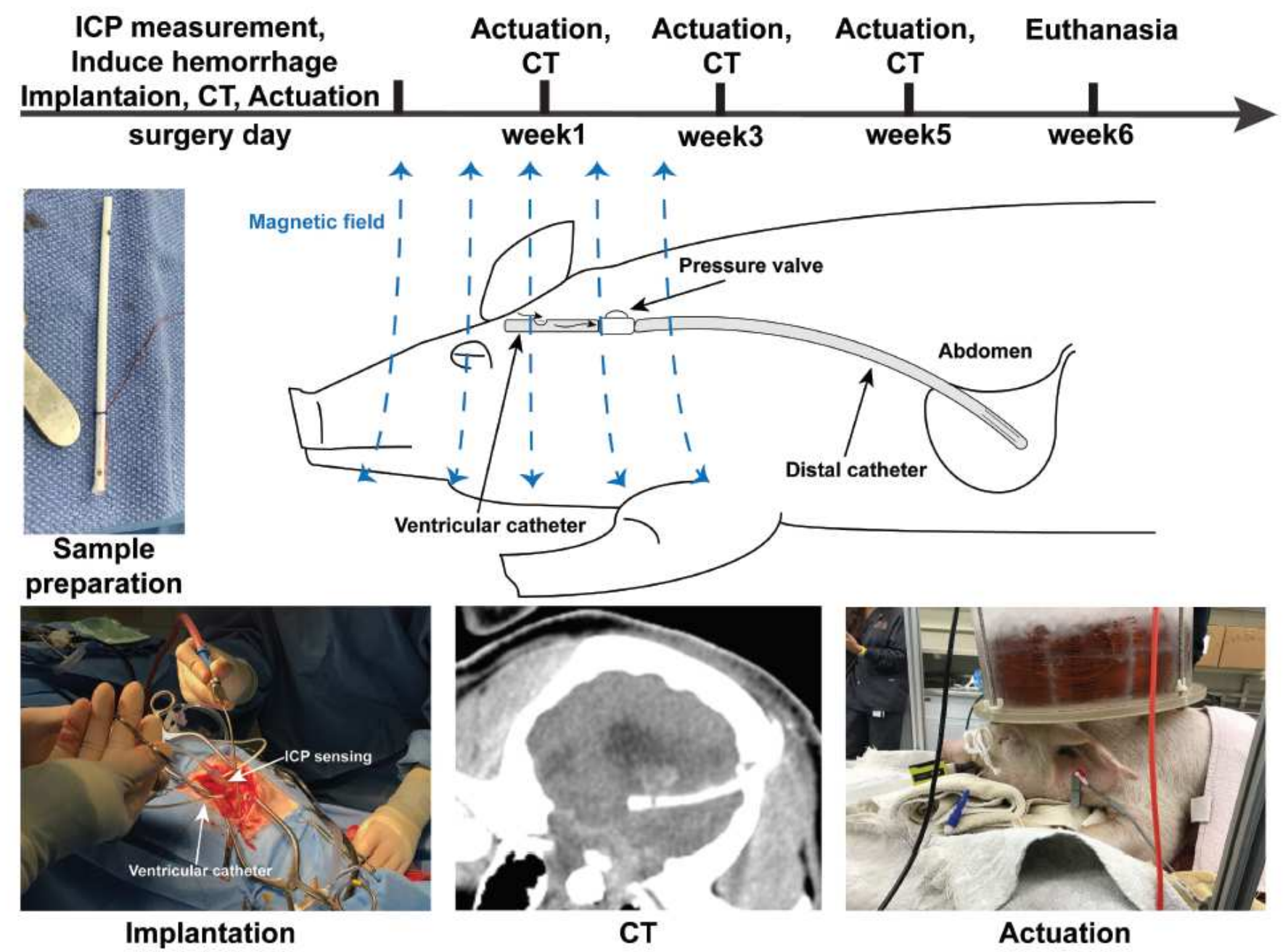

Figure 4

In vivo experimental timeline. The entire study is designed to lasts 6 weeks. On surgery day, we induced IVH and implanted a VP shunt. CT was performed before and after the surgical procedure, to measure the ventricular size and confirm ventricular catheter placement. Magnetic actuation (30 min) was performed for the Treated group immediately afterward. At weeks 1, 3, and 5, each surviving animal was subjected to 30 min of magnetic field and CT scans. All surviving animals were terminated at week 6 for explantation and necropsy. 


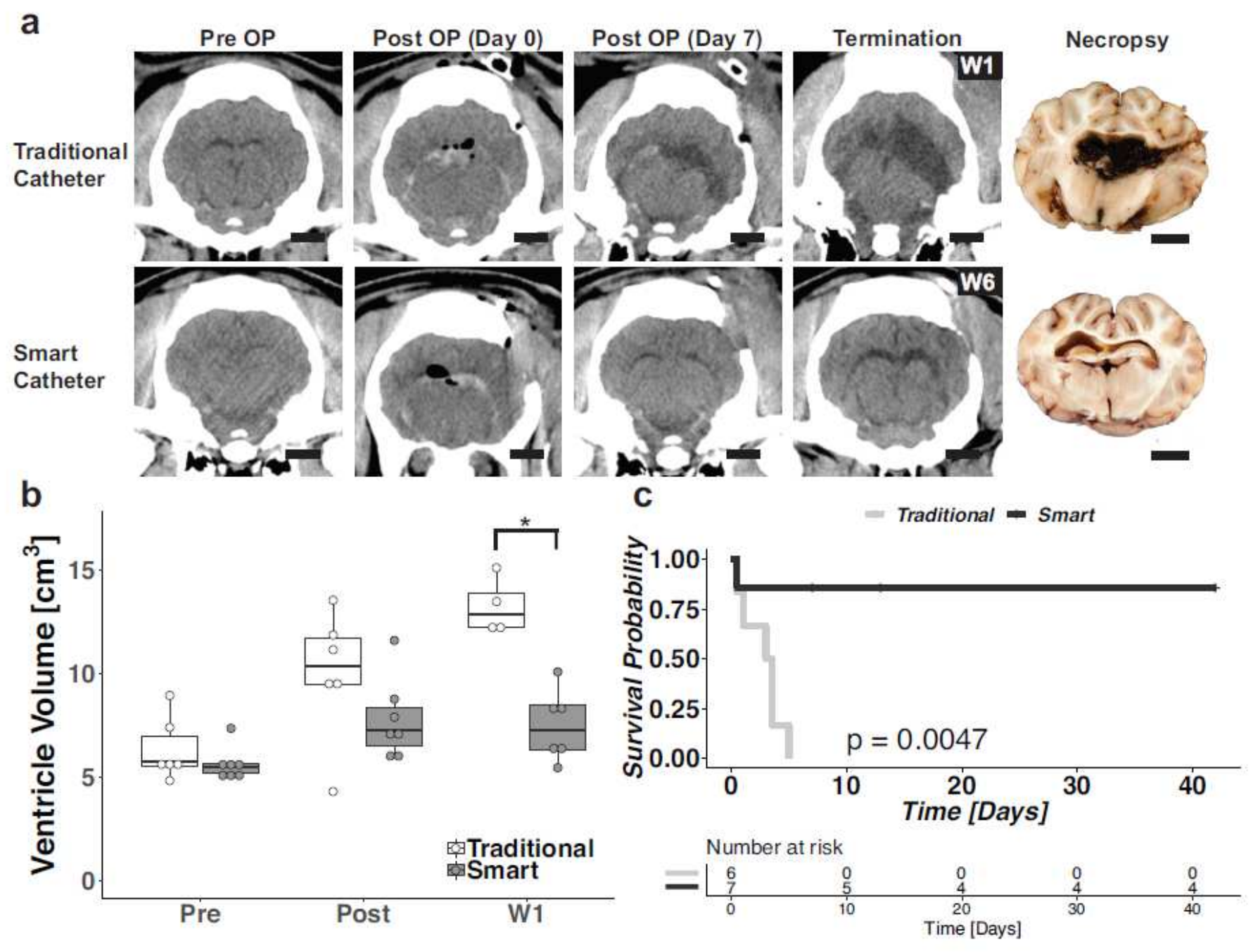

\section{Figure 5}

In vivo evaluation results. (a) Comparison of CT scans over the course of implantation for animals with traditional catheters (Control) and smart self-clearing catheters (Treated). Note the difference in ventricular volume before and after the surgery. There are signs of air pockets following the surgery (Post Op). After a week, the air pockets are resorbed but there is significant evidence of ventriculomegaly and hematoma in Control animals. The CT scan of the Control animal shows a significantly larger ventricle filled with hematoma compared to the Treatment animal. The photograph at necropsy also shows the enlarged ventricles. Scale bars $=1 \mathrm{~cm}$. (b) Box plot of ventricle volume until week 1 . The extended ventricle volume plot is available in SI Fig. 6. ANOVA indicated a significant difference in ventricle volume when treated with smart self-clearing catheters. Control animals with traditional catheters had a significant increase in ventricle volume by W1 ( $p<0: 001)$. (c) Kaplan-Meier survival plot with corresponding risk table. By week 1 , the traditional shunt systems in all Control animals had failed whereas $80 \%$ of the shunt systems with self-clearing catheters remained obstruction-free with biweekly actuation. SI Fig. 7 shows the survival plot when infections are counted as failures. 


\section{Supplementary Files}

This is a list of supplementary files associated with this preprint. Click to download.

- 2020HLeeSIFinal.pdf

- SIVideo1.mp4

- SIVideo2.mp4

- SIVideo3.mp4 